\title{
Convergence theorems for split equality generalized mixed equilibrium problems for demi-contractive mappings
}

\author{
Mijanur Rahaman'1 Yeong-Cheng Liou ${ }^{2,3^{*}}$, Rais Ahmad' and lqbal Ahmad ${ }^{1}$
}

"Correspondence:
simplex_liou@hotmail.com
Department of Information
Management, Cheng Shiu
University, Kaohsiung, 833, Taiwan
${ }^{3}$ Research Center of Nonlinear
Analysis and Optimization and
Center for Fundamental Science,
Kaohsiung Medical University,
Kaohsiung, 807, Taiwan
Full list of author information is
available at the end of the article

available at the end of the article

\begin{abstract}
In this paper, we introduce a new iterative algorithm for solving the split equality generalized mixed equilibrium problems. The weak and strong convergence theorems are proved for demi-contractive mappings in real Hilbert spaces. Several special cases are also discussed. As applications, we employ our results to get the convergence results for the split equality convex differentiable optimization problem, the split equality convex minimization problem, and the split equality mixed equilibrium problem. The results in this paper generalize, extend, and unify some recent results in the literature.
\end{abstract}

MSC: 47H09; 47J25; 49M37; 90C25

Keywords: split equality generalized mixed equilibrium problem; demi-contractive mappings; fixed point; iterative scheme

\section{Introduction}

The equilibrium problem has been extensively studied, beginning with Blum and Oettli [1] where they proposed it as a generalization of optimization and variational inequality problem. The classical, scalar-valued equilibrium problem deals with the existence of $x^{*} \in$ $C$ such that

$$
F\left(x^{*}, y\right) \geq 0, \quad \forall y \in C
$$

where $C$ is the nonempty closed convex subset of a real Hilbert space $H$ and $F: C \times C \longrightarrow$ $\mathbb{R}$ is a bi-mapping.

Very recently, Ahmad and Rahaman [2] introduced the generalized vector equilibrium problem of finding $x \in C$ such that

$$
F(\lambda x+(1-\lambda) z, y) \nsubseteq \subseteq-C \backslash\{0\}, \quad \forall y, z \in C, \lambda \in(0,1],
$$

where $F: C \times C \longrightarrow 2^{H}$ is the set-valued mapping with the condition $F(\lambda x+(1-\lambda) z, x) \supseteq$ $\{0\}$, and $[\cdot, z)$ denotes the line-segment excluding the point $z$. In the scalar case, the generalized equilibrium problem takes the form to find $x^{*} \in C$ such that

$$
F\left(\lambda x^{*}+(1-\lambda) z, y\right) \geq 0, \quad \forall y, z \in C, \lambda \in(0,1]
$$

(c) 2015 Rahaman et al. This article is distributed under the terms of the Creative Commons Attribution 4.0 International License (http://creativecommons.org/licenses/by/4.0/), which permits unrestricted use, distribution, and reproduction in any medium, provided you give appropriate credit to the original author(s) and the source, provide a link to the Creative Commons license, and indicate if changes were made. 
with the condition $F(\lambda x+(1-\lambda) z, x)=0$. If $\lambda=1$, then the generalized equilibrium problem (1.2) reduces to the classical equilibrium problem (1.1).

In 1999, Moudafi and Thèra [3] introduced the mixed equilibrium problem of finding $x^{*} \in C$ such that

$$
F\left(x^{*}, y\right)+\left\langle T\left(x^{*}\right), y-x^{*}\right\rangle \geq 0, \quad \forall y \in C,
$$

where $F: C \times C \longrightarrow \mathbb{R}$ is a given bi-mapping with $F(x, x)=0$, for all $x \in C$ and $T: C \longrightarrow$ $C$ is a continuous mapping. Problem (1.3) has useful applications in nonlinear analysis, including optimization problems, variational inequalities, fixed-point problems, and the problems of Nash equilibria as special cases.

In 2008, Peng and Yao [4] considered the following extended mixed equilibrium problem (EMEP): Find $x^{*} \in C$ such that

$$
F\left(x^{*}, y\right)+\left\langle T\left(x^{*}\right), y-x^{*}\right\rangle+\phi(y)-\phi\left(x^{*}\right) \geq 0, \quad \forall y \in C,
$$

where $\phi: C \longrightarrow \mathbb{R} \cup\{+\infty\}$ is a mapping.

If $T=0$, then the extended mixed equilibrium problem (1.4) becomes the mixed equilibrium problem to find $x^{*} \in C$ such that

$$
F\left(x^{*}, y\right)+\phi(y)-\phi\left(x^{*}\right) \geq 0, \quad \forall y \in C .
$$

Problem (1.5) was studied by Ceng and Yao [5]. If $T=0$ and $F(x, y)=0$, for all $x, y \in C$, the extended mixed equilibrium problem (1.4) becomes the following convex minimization problem:

$$
\text { Finding } x^{*} \in C \quad \text { such that } \quad \phi(y)-\phi\left(x^{*}\right) \geq 0, \quad \forall y \in C .
$$

Now, we mention the following generalized mixed equilibrium problem of finding $x^{*} \in C$ :

$$
F\left(\lambda x^{*}+(1-\lambda) b, y\right)+\left\langle T\left(x^{*}\right), y-x^{*}\right\rangle+\phi(y)-\phi\left(x^{*}\right) \geq 0, \quad \forall y, b \in C, \lambda \in(0,1] .
$$

The solution set of problem (1.7) is denoted by $\operatorname{GMEP}(F, T, \phi)$.

The convex feasibility problem (CFP) considered in [6] is a central problem in applied mathematics, which can be formulated in various ways to find a common point of closed and convex sets, to find a common fixed-point of nonexpansive mappings, to find a common minimum of convex functionals, to solve a system of variational inequalities. The (CFP) is to find a member of the intersection of finitely many closed convex sets in Euclidean spaces. When the intersection is empty, one can minimize a proximity function to obtain an approximate solution to the problem. The split feasibility problem (SFP) and the split equality problem (SEP) are generalizations of the (CFP). The (SFP) arises in many areas of applications such as phase retrieval, medical image reconstruction, computer temography and radiation therapy treatment planning; see, e.g., [7-11]. In 1994, Censor and Elfving [10] introduced the following (SFP) in finite dimensional Hilbert spaces. 
Let $H_{1}, H_{2}$, and $H_{3}$ be the real Hilbert spaces. Let $C$ and $Q$ be two nonempty closed convex subsets of real Hilbert spaces $H_{1}$ and $H_{2}$, respectively, $A: H_{1} \longrightarrow H_{2}$ be a bounded linear mapping. The (SFP) is to find a point $x^{*}$ such that

$$
x^{*} \in C \text { and } A x^{*} \in Q .
$$

Recently, Moudafi and Al-Shemas [12] introduced the split equality problem (SEP) of finding $x^{*}$ and $y^{*}$ with the property

$$
x^{*} \in C, y^{*} \in Q \quad \text { such that } A x^{*}=B y^{*} \text {, }
$$

where $A: H_{1} \longrightarrow H_{3}$ and $B: H_{2} \longrightarrow H_{3}$ are bounded linear mappings, which allows for asymmetric and partial relations between the variables $x^{*}$ and $y^{*}$. If $H_{2}=H_{3}$ and $B=I$, then the (SEP) (1.9) reduces to the (SFP) (1.8).

In order to study the weak convergence properties of (SEP) (1.9), Moudafi and AlShemas [12] introduced the following simultaneous iterative method:

$$
\left\{\begin{array}{l}
x_{n+1}=U\left(x_{n}-\gamma_{n} A^{*}\left(A x_{n}-B y_{n}\right)\right) \\
y_{n+1}=T\left(y_{n}+\gamma_{n} B^{*}\left(A x_{n}-B y_{n}\right)\right)
\end{array}\right.
$$

where $U: H_{1} \longrightarrow H_{1}, T: H_{2} \longrightarrow H_{2}$ are firmly quasi-nonexpansive mappings, $A^{*}$ and $B^{*}$ are the adjoint of $A$ and $B$, respectively. Under some suitable conditions, they proved the weak convergence result for the SEP (1.9).

To get the strong and weak convergence theorems for the (SEP) (1.9), Ma et al. [13] generalized the corresponding results of Moudafi and Al-Shemas [12], and they introduced the following iterative algorithm under some mild control conditions in Hilbert spaces:

$$
\left\{\begin{array}{l}
x_{n+1}=\left(1-\alpha_{n}\right) x_{n}+\alpha_{n} U\left(x_{n}-\gamma_{n} A^{*}\left(A x_{n}-B y_{n}\right)\right) ; \\
y_{n+1}=\left(1-\alpha_{n}\right) y_{n}+\alpha_{n} T\left(y_{n}-\gamma_{n} B^{*}\left(A x_{n}-B y_{n}\right)\right),
\end{array}\right.
$$

where $\left\{\alpha_{n}\right\} \subset[\alpha, 1]$, for some $\alpha>0$.

Recently, He [14] introduced the following split equilibrium problem ( $\left.\mathrm{S} E_{q} \mathrm{P}\right)$. Let $F: C \times$ $C \longrightarrow \mathbb{R}$ and $G: Q \times Q \longrightarrow \mathbb{R}$ be two bi-mappings, $A: H_{1} \longrightarrow H_{2}$ be a bounded linear mapping. The split equilibrium problem is to find an element $x^{*} \in C$ such that

$$
F\left(x^{*}, x\right) \geq 0, \quad \forall x \in C,
$$

and such that

$$
y^{*}=A x^{*} \in Q \quad \text { solves } \quad G\left(y^{*}, y\right) \geq 0, \quad \forall y \in Q .
$$

To solve the split equilibrium problem $\left(S E_{q} \mathrm{P}\right), \mathrm{He}$ [14] proposed the following iterative algorithm:

$$
\begin{cases}F\left(u_{n}, u\right)+\frac{1}{r_{n}}\left\langle u-u_{n}, u_{n}-x_{n}\right\rangle \geq 0, \quad \forall u \in C ; \\ G\left(v_{n}, v\right)+\frac{1}{r_{n}}\left\langle v-v_{n}, v_{n}-A u_{n}\right\rangle \geq 0, \quad \forall v \in Q ; \\ x_{n+1}=P_{C}\left(u_{n}\right)+\mu A^{*}\left(v_{n}-A u_{n}\right),\end{cases}
$$


where $P_{C}$ is the metric projection operator from $H_{1}$ onto $C, r_{n} \subset(0, \infty)$ with $\lim _{n \rightarrow \infty} \inf r_{n}>0$, and $\mu \in\left(0, \frac{1}{\left\|A^{*}\right\|^{2}}\right)$ is a constant.

Very recently, Ma et al. [15] considered the following split equality mixed equilibrium problem (SEMEP). Let $\phi: C \longrightarrow \mathbb{R} \cup\{+\infty\}$ and $\varphi: Q \longrightarrow \mathbb{R} \cup\{+\infty\}$ be proper lower semicontinuous and convex mappings such that $C \cap \operatorname{dom} \phi \neq \emptyset$ and $Q \cap \operatorname{dom} \varphi \neq \emptyset$, and $A: H_{1} \longrightarrow H_{3}$ and $B: H_{2} \longrightarrow H_{3}$ be bounded linear mappings. Then the SEMEP is to find $x^{*} \in C$ and $y^{*} \in Q$ such that

$$
\begin{aligned}
& F\left(x^{*}, x\right)+\phi(x)-\phi\left(x^{*}\right) \geq 0, \quad \forall x \in C, \\
& G\left(y^{*}, y\right)+\varphi(y)-\varphi\left(y^{*}\right) \geq 0, \quad \forall y \in Q, \quad \text { and } \\
& A x^{*}=B y^{*} .
\end{aligned}
$$

The set of solutions of (1.15) is denoted by $\operatorname{SEMEP}(F, G, \phi, \varphi)$.

In order to obtain the weak and strong convergence results of (SEMEP) (1.15), Ma et al. [15] presented the following simultaneous iterative algorithm:

$$
\left\{\begin{array}{l}
F\left(u_{n}, u\right)+\phi(u)-\phi\left(u_{n}\right)+\frac{1}{r_{n}}\left\langle u-u_{n}, u_{n}-x_{n}\right\rangle \geq 0, \quad \forall u \in C ; \\
G\left(v_{n}, v\right)+\varphi(v)-\varphi\left(v_{n}\right)+\frac{1}{r_{n}}\left\langle v-v_{n}, v_{n}-A u_{n}\right\rangle \geq 0, \quad \forall v \in Q ; \\
x_{n+1}=\alpha_{n} u_{n}+\left(1-\alpha_{n}\right) T\left(u_{n}-\gamma_{n} A^{*}\left(A u_{n}-B v_{n}\right)\right) ; \\
y_{n+1}=\alpha_{n} v_{n}+\left(1-\alpha_{n}\right) S\left(v_{n}+\gamma_{n} B^{*}\left(A u_{n}-B v_{n}\right)\right),
\end{array}\right.
$$

where $T: H_{1} \longrightarrow H_{1}, S: H_{2} \longrightarrow H_{2}$ are nonexpansive mappings.

In this paper, we consider the following split equality generalized mixed equilibrium problem (SEGMEP).

Let $F: C \times C \longrightarrow \mathbb{R}$ and $G: Q \times Q \longrightarrow \mathbb{R}$ be two nonlinear bi-mappings, $T: C \longrightarrow C$ and $S: Q \longrightarrow Q$ be two nonlinear mappings, and $\phi: C \longrightarrow \mathbb{R} \cup\{+\infty\}$ and $\varphi: Q \rightarrow \mathbb{R} \cup$ $\{+\infty\}$ be proper lower semicontinuous and convex mappings such that $C \cap \operatorname{dom} \phi \neq \emptyset$ and $Q \cap \operatorname{dom} \varphi \neq \emptyset$. Let $A: H_{1} \longrightarrow H_{3}$ and $B: H_{2} \longrightarrow H_{3}$ be bounded linear mappings. Then the split equality generalized mixed equilibrium problem (SEGMEP) is to find $x^{*} \in C$ and $y^{*} \in Q$ such that

$$
\begin{aligned}
& \quad F\left(\lambda_{1} x^{*}+\left(1-\lambda_{1}\right) b, x\right)+\left\langle T\left(x^{*}\right), x-x^{*}\right\rangle+\phi(x)-\phi\left(x^{*}\right) \geq 0, \\
& \quad \forall x, b \in C, \lambda_{1} \in(0,1], \\
& G\left(\lambda_{2} y^{*}+\left(1-\lambda_{2}\right) c, y\right)+\left\langle S\left(y^{*}\right), y-y^{*}\right\rangle+\varphi(y)-\varphi\left(y^{*}\right) \geq 0, \\
& \quad \forall y, c \in Q, \lambda_{2} \in(0,1], \quad \text { and } \\
& A x^{*}=B y^{*} .
\end{aligned}
$$

The solution set of problem (1.17) is denoted by $\operatorname{SEMEP}(F, G, T, S, \phi, \varphi)$.

Special cases:

(1) If $\lambda_{1}=\lambda_{2}=1$ and $T=S=0$, then the split equality generalized mixed equilibrium problem (1.17) becomes the split equality mixed equilibrium problem (1.15).

(2) If $\lambda_{1}=\lambda_{2}=1, T=S=0$, and $\phi=\varphi=0$, then the split equality generalized mixed equilibrium problem (1.17) reduces to the following split equality equilibrium 
problem: find $x^{*} \in C$ and $y^{*} \in Q$ such that

$$
F\left(x^{*}, x\right) \geq 0, \quad \forall x \in C, \quad G\left(y^{*}, y\right) \geq 0, \quad \forall y \in Q, \quad \text { and } \quad A x^{*}=B y^{*} .
$$

The set of solutions of $(1.18)$ is denoted by $\operatorname{SEEP}(F, G)$.

(3) If $F=G=0$ and $T=S=0$, then the split equality generalized mixed equilibrium problem (1.17) becomes the split equality convex minimization problem to find $x^{*} \in C$ and $y^{*} \in Q$ such that

$$
\phi(x) \geq \phi\left(x^{*}\right), \quad \forall x \in C, \quad \varphi(y) \geq \varphi\left(y^{*}\right), \quad \forall y \in Q, \quad \text { and } \quad A x^{*}=B y^{*} .
$$

The set of solutions of (1.19) is denoted by $\operatorname{SECMP}(\phi, \varphi)$.

(4) If $B=I$, then the split equality convex minimization problem (1.19) reduces to the following split convex minimization problem. Find $x^{*} \in C$ such that

$$
\begin{aligned}
& \phi(x) \geq \phi\left(x^{*}\right), \quad \forall x \in C, \quad \text { and } \\
& y^{*}=A x^{*} \in Q \quad \text { such that } \quad \varphi(y) \geq \varphi\left(y^{*}\right), \quad \forall y \in Q .
\end{aligned}
$$

The set of solutions of (1.20) is denoted by $\operatorname{SCMP}(\phi, \varphi)$.

In this paper, by using the well-known KKM technique, we derive an important lemma which is a foundation for studying the generalized mixed equilibrium problem (1.7). Motivated by the recent above work of Moudafi et al. [12], Ma et al. [15], Ma et al. [13] and Chidume et al. [16], in this paper, we introduce a new iterative algorithm for solving the split equality generalized mixed equilibrium problem (1.17) for demi-contractive mappings. We obtain weak and strong convergence results for the sequences generated by these processes. As applications, we employ our results to study the convergence results for the split equality convex differentiable optimization problem, the split equality convex minimization problem, and the split equality mixed equilibrium problem. The results of this paper generalize, extend, and unify some well-known weak and strong convergence results in the literature mentioned above.

\section{Preliminaries}

We first recall some definitions and known results which are needed to prove our main results.

In the sequel, let $H$ be a real Hilbert space with inner product $\langle\cdot, \cdot\rangle$, and norm $\|\cdot\|$. Let $C$ be a nonempty closed convex subset of $H$. Let the symbols $\rightarrow$ and $\rightarrow$ denote strong and weak convergence, respectively. A point $x \in H$ is said to be a fixed point of $T$ provided $T x=x$, where $T: H \longrightarrow H$ is a mapping. We denote the set of fixed points of the mapping $T$ by $\operatorname{Fix}(T)$. It is well known that

$$
\|\lambda x+(1-\lambda) y\|^{2}=\lambda\|x\|^{2}+(1-\lambda)\|y\|^{2}-\lambda(1-\lambda)\|x-y\|^{2},
$$

for all $x, y \in H$ and $\lambda \in[0,1]$.

Definition 2.1 A mapping $T: H \longrightarrow H$ is said to be firmly quasi-nonexpansive if $\operatorname{Fix}(T) \neq$ $\emptyset$ and

$$
\left\|T x-x^{*}\right\|^{2} \leq\left\|x-x^{*}\right\|^{2}-\|x-T x\|^{2}, \quad \forall x \in H, x^{*} \in \operatorname{Fix}(T) .
$$


Definition 2.2 Let $C$ be a nonempty subset of $H$. The mapping $T: C \longrightarrow C$ is said to be $k$-demi-contractive if $\operatorname{Fix}(T) \neq \emptyset$ and there exists a constant $k \in(0,1)$ such that

$$
\left\|T x-x^{*}\right\|^{2} \leq\left\|x-x^{*}\right\|^{2}+k\|x-T x\|^{2}, \quad \forall x \in C, x^{*} \in \operatorname{Fix}(T)
$$

Remark 2.1 Clearly, the class of firmly quasi-nonexpansive mappings is a subclass of demi-contractive mappings. Note also that the mapping $T$ satisfying (2.2) with $k=1$ is usually called hemicontractive. It is easy to observe from (2.2) that

$$
\begin{aligned}
\left\|T x-x^{*}\right\| & \leq\left\|x-x^{*}\right\|+\sqrt{k}\|x-T x\| \\
& \leq(1+\sqrt{k})\left\|x-x^{*}\right\|+\sqrt{k}\left\|T x-x^{*}\right\| \\
& =\left(\frac{1+\sqrt{k}}{1-\sqrt{k}}\right)\left\|x-x^{*}\right\| \\
& =L\left\|x-x^{*}\right\|,
\end{aligned}
$$

where $L=\frac{1+\sqrt{k}}{1-\sqrt{k}}$, so that

$$
\left\|T x-x^{*}\right\| \leq L\left\|x-x^{*}\right\| .
$$

Definition 2.3 A mapping $T: C \longrightarrow H$ is said to be demi-closed at a point $z \in H$ if, the weak convergence of $\left\{x_{n}\right\}$ in $C$ to some point $p \in C$ and the strong convergence of $\left\{T x_{n}\right\}$ to $z$ implies that $T p=z$.

Definition 2.4 A mapping $T: C \longrightarrow H$ is said to be demi-compact at a point $z \in H$ if, for any bounded sequence $\left\{x_{n}\right\}$ in $C$ such that $(I-T) x_{n} \rightarrow z$ as $n \rightarrow \infty$, then there exist a subsequence $\left\{x_{n_{j}}\right\}$ and a point $p \in C$ such that $x_{n_{j}} \rightarrow p$ as $j \rightarrow \infty$ and $(I-T) p=z$.

Definition 2.5 A multi-valued mapping $F: C \longrightarrow 2^{H}$ is said to be KKM-mapping if, for each finite subset $\left\{x_{1}, \ldots, x_{n}\right\}$ of $C, \operatorname{Co}\left\{x_{1}, \ldots, x_{n}\right\} \subseteq \bigcup_{i=1}^{n} F\left(x_{i}\right)$, where $\operatorname{Co}\left\{x_{1}, \ldots, x_{n}\right\}$ denotes the convex hull of $\left\{x_{1}, \ldots, x_{n}\right\}$.

Theorem 2.1 (KKM theorem [17]) Let $C$ be a subset of a Hausdorff topological vector space $H$ and let $F: C \longrightarrow 2^{H}$ be a KKM-mapping. Iffor each $x \in C, F(x)$ is closed and iffor at least one point $x \in C, F(x)$ is compact, then $\bigcap_{x \in C} F(x) \neq \emptyset$.

Lemma 2.1 ([18]) Let $C$ be a nonempty closed convex subset of a strictly convex Banach space $X$, and $T: C \longrightarrow C$ be a nonexpansive mapping with $\operatorname{Fix}(T) \neq \emptyset$. Then $\operatorname{Fix}(T)$ is closed and convex.

For solving the generalized mixed equilibrium problem (1.7), let us give the following assumptions for the bi-mapping $F: C \times C \longrightarrow \mathbb{R}$, and the mapping $T: C \longrightarrow C$ :

(A1) $F(\lambda x+(1-\lambda) b, x)=0$, for all $x \in C$;

(A2) $F$ is monotone, i.e., $F(\lambda x+(1-\lambda) b, y)+F(\lambda y+(1-\lambda) b, x) \leq 0$, for all $x, y \in C$;

(A3) $T$ is monotone, i.e., $\langle T(x)-T(y), x-y\rangle \geq 0$, for all $x, y \in C$;

(A4) for each $x \in C, y \mapsto F(\lambda x+(1-\lambda) b, y)$ is convex and lower semicontinuous;

(A5) $F$ is hemicontinuous in the first argument; 
(A6) $T$ is weakly upper semicontinuous;

(A7) for each $x \in C, \lambda \in(0,1]$ and $r>0$, there exist a bounded set $D \subset C$ and $a \in C$ such that for any $z \in C \backslash D$,

$$
-F(\lambda a+(1-\lambda) b, z)+\langle T(z), a-z\rangle+\phi(a)-\phi(z)+\frac{1}{r}\langle a-z, z-x\rangle<0, \quad \forall b \in C .
$$

Lemma 2.2 Let $C$ be a nonempty closed convex subset of a Hilbert space $H_{1}$. Suppose that the bi-mapping $F: C \times C \longrightarrow \mathbb{R}$ and the mapping $T: C \longrightarrow C$ satisfy the conditions (A1)(A7). Let $\phi: C \longrightarrow \mathbb{R} \cup\{+\infty\}$ be a proper lower semicontinuous and convex mapping such that $C \cap \operatorname{dom} \phi \neq \emptyset$. For $r>0, \lambda_{1} \in(0,1]$ and $x \in H_{1}$, let $J_{r}^{F, T}: H_{1} \rightarrow C$ be the resolvent operator of $F$ and $T$, defined by

$$
\begin{aligned}
J_{r}^{F, T}(x)= & \left\{z \in C: F\left(\lambda_{1} z+\left(1-\lambda_{1}\right) b, y\right)+\langle T(z), y-z\rangle\right. \\
& \left.+\phi(y)-\phi(z)+\frac{1}{r}\langle y-z, z-x\rangle \geq 0, \forall y, b \in C\right\} .
\end{aligned}
$$

Then:

(i) for each $x \in H_{1}, J_{r}^{F, T}(x) \neq \emptyset$;

(ii) $J_{r}^{F, T}$ is single-valued;

(iii) $J_{r}^{F, T}$ is firmly nonexpansive, i.e., for any $x, y \in H_{1}$,

$$
\left\|J_{r}^{F, T}(x)-J_{r}^{F, T}(y)\right\|^{2} \leq\left\langle J_{r}^{F, T}(x)-J_{r}^{F, T}(y), x-y\right\rangle
$$

(iv) $\operatorname{Fix}\left(J_{r}^{F, T}\right)=\operatorname{GMEP}(F, T, \phi)$ and it is closed and convex.

Proof (i) Let $\bar{x}$ be any given point in $H_{1}$. For each $y \in C$, we define

$$
\begin{aligned}
M(y)= & \left\{z \in C:-F\left(\lambda_{1} y+\left(1-\lambda_{1}\right) b, z\right)+\langle T(z), y-z\rangle\right. \\
& \left.+\phi(y)-\phi(z)+\frac{1}{r}\langle y-z, z-\bar{x}\rangle \geq 0\right\} .
\end{aligned}
$$

Since $y \in M(y), M(y)$ is nonempty. Now, we show that $M$ is a KKM-mapping. To the contrary, suppose that $M$ is not a KKM-mapping. Then there exist a finite subset $\left\{y_{1}, \ldots, y_{n}\right\}$ of $C$ and $t_{i} \geq 0$ for all $i=1, \ldots, n$ with $\sum_{i=1}^{n} t_{i}=1$ such that $\bar{z}=\sum_{i=1}^{n} t_{i} y_{i} \notin \bigcup_{i=1}^{n} M\left(y_{i}\right)$, for each $i$. Then we have

$$
-F\left(\lambda_{1} y_{i}+\left(1-\lambda_{1}\right) b, \bar{z}\right)+\left\langle T(\bar{z}), y_{i}-\bar{z}\right\rangle+\phi\left(y_{i}\right)-\phi(\bar{z})+\frac{1}{r}\left\langle y_{i}-\bar{z}, \bar{z}-\bar{x}\right\rangle<0, \quad \forall i
$$

By using (A1)-(A4) and convexity of $\phi$, we have

$$
\begin{aligned}
0 & =F\left(\lambda_{1} \bar{z}+\left(1-\lambda_{1}\right) b, \bar{z}\right)+\langle T(\bar{z}), \bar{z}-\bar{z}\rangle+\phi(\bar{z})-\phi(\bar{z})+\frac{1}{r}\langle\bar{z}-\bar{z}, \bar{z}-\bar{x}\rangle \\
& =F\left(\lambda_{1} \bar{z}+\left(1-\lambda_{1}\right) b, \sum_{i=1}^{n} t_{i} y_{i}\right)+\left\langle T(\bar{z}), \sum_{i=1}^{n} t_{i} y_{i}-\sum_{i=1}^{n} t_{i} \bar{z}\right\rangle+\phi\left(\sum_{i=1}^{n} t_{i} y_{i}\right)
\end{aligned}
$$




$$
\begin{aligned}
& -\phi(\bar{z})+\frac{1}{r}\left\langle\sum_{i=1}^{n} t_{i} y_{i}-\bar{z}, \bar{z}-\bar{x}\right\rangle \\
\leq & \sum_{i=1}^{n} t_{i} F\left(\lambda_{1} \bar{z}+\left(1-\lambda_{1}\right) b, y_{i}\right)+\sum_{i=1}^{n} t_{i}\left\langle T(\bar{z}), y_{i}-\bar{z}\right\rangle+\sum_{i=1}^{n} t_{i} \phi\left(y_{i}\right)-\sum_{i=1}^{n} t_{i} \phi(\bar{z}) \\
& +\frac{1}{r} \sum_{i=1}^{n} t_{i}\left\langle y_{i}-\bar{z}, \bar{z}-\bar{x}\right\rangle \\
\leq & -\sum_{i=1}^{n} t_{i} F\left(\lambda_{1} y_{i}+\left(1-\lambda_{1}\right) b, \bar{z}\right) \\
& +\sum_{i=1}^{n} t_{i}\left\{\left\langle T(\bar{z}), y_{i}-\bar{z}\right\rangle+\phi\left(y_{i}\right)-\phi(\bar{z})+\frac{1}{r}\left\langle y_{i}-\bar{z}, \bar{z}-\bar{x}\right\rangle\right\} \\
= & \sum_{i=1}^{n} t_{i}\left\{-F\left(\lambda_{1} y_{i}+\left(1-\lambda_{1}\right) b, \bar{z}\right)+\left\langle T(\bar{z}), y_{i}-\bar{z}\right\rangle+\phi\left(y_{i}\right)-\phi(\bar{z})+\frac{1}{r}\left\langle y_{i}-\bar{z}, \bar{z}-\bar{x}\right\rangle\right\} \\
< & 0,
\end{aligned}
$$

which is not possible, and hence $M$ is a KKM-mapping.

Now, we prove that $M(y)=\overline{M(f)}^{w}$ for each $y \in C$, i.e., $M(y)$ is weakly closed. Let $z \in$ $\overline{M(f)}^{w}$ and $\left\{z_{n}\right\}$ be a sequence in $M(y)$ such that $z_{n} \rightarrow z$. Since $z_{n} \in M(y)$, we have

$$
-F\left(\lambda_{1} y+\left(1-\lambda_{1}\right) b, z_{n}\right)+\left\langle T\left(z_{n}\right), y-z_{n}\right\rangle+\phi(y)-\phi\left(z_{n}\right)+\frac{1}{r}\left\langle y-z_{n}, z_{n}-\bar{x}\right\rangle \geq 0 .
$$

It follows from (A4) and (A6), and the weak lower semicontinuity of $\phi$ and $\|\cdot\|^{2}$ that

$$
\begin{aligned}
0 \leq & \limsup _{n \rightarrow \infty}\left\{-F\left(\lambda_{1} y+\left(1-\lambda_{1}\right) b, z_{n}\right)+\left\langle T\left(z_{n}\right), y-z_{n}\right\rangle+\phi(y)-\phi\left(z_{n}\right)+\frac{1}{r}\left\langle y-z_{n}, z_{n}-\bar{x}\right\rangle\right\} \\
\leq & \limsup _{n \rightarrow \infty}\left\{-F\left(\lambda_{1} y+\left(1-\lambda_{1}\right) b, z_{n}\right)\right\}+\limsup _{n \rightarrow \infty}\left\{\left\langle T\left(z_{n}\right), y-z_{n}\right\rangle+\phi(y)\right\} \\
& -\liminf _{n \rightarrow \infty} \phi\left(z_{n}\right)+\frac{1}{r} \limsup _{n \rightarrow \infty}\left\langle y-z_{n}, z_{n}-\bar{x}\right\rangle \\
\leq & -\liminf _{n \rightarrow \infty}\left\{F\left(\lambda_{1} y+\left(1-\lambda_{1}\right) b, z_{n}\right)\right\}+\limsup _{n \rightarrow \infty}\left\{\left\langle T\left(z_{n}\right), y-z_{n}\right\rangle+\phi(y)\right\} \\
& -\liminf _{n \rightarrow \infty} \phi\left(z_{n}\right)+\frac{1}{r} \limsup _{n \rightarrow \infty}\left\langle y-z_{n}, z_{n}-\bar{x}\right\rangle \\
\leq & -F\left(\lambda_{1} y+\left(1-\lambda_{1}\right) b, z\right)+\langle T(z), y-z\rangle+\phi(y)-\phi(z)+\frac{1}{r}\langle y-z, z-\bar{x}\rangle .
\end{aligned}
$$

This implies that $z \in M(y)$, and hence $M(y)$ is weakly closed.

In order to show that $M(y)$ is weakly compact for at least a point $y \in C$, from (A7), we can see that there exist a bounded set $D \subset C$ and $a \in D$ such that for any $z \in C \backslash D$, we have $z \notin M(a)$. Then $M(a) \subset D$, i.e., it is bounded, which shows that $M(y)$ is weakly compact. Then by KKM theorem 2.1, $\bigcap_{y \in C} M(y) \neq \emptyset$. Hence, for $z \in \bigcap_{y \in C} M(y)$, we have

$$
-F\left(\lambda_{1} y+\left(1-\lambda_{1}\right) b, z\right)+\langle T(z), y-z\rangle+\phi(y)-\phi(z)+\frac{1}{r}\langle y-z, z-\bar{x}\rangle \geq 0
$$


Let $y \in C$ be arbitrary, and let $z_{t}=t y+(1-t) z, 0<t \leq 1$. Then $z_{t} \in C$, and hence we have

$$
-F\left(\lambda_{1} z_{t}+\left(1-\lambda_{1}\right) b, z\right)+\left\langle T(z), z_{t}-z\right\rangle+\phi\left(z_{t}\right)-\phi(z)+\frac{1}{r}\left\langle z_{t}-z, z-\bar{x}\right\rangle \geq 0 .
$$

Applying (2.6), using (A1), (A4), and convexity of $\phi$, we have

$$
\begin{aligned}
0= & F\left(\lambda_{1} z_{t}+(1-\lambda) b, z_{t}\right) \\
= & F\left(\lambda_{1} z_{t}+(1-\lambda) b, t y+(1-t) z\right) \\
\leq & t F\left(\lambda_{1} z_{t}+(1-\lambda) b, y\right)+(1-t) F\left(\lambda_{1} z_{t}+(1-\lambda) b, z\right) \\
\leq & t F\left(\lambda_{1} z_{t}+(1-\lambda) b, y\right)+(1-t)\left\{\left\langle T(z), z_{t}-z\right\rangle+\phi\left(z_{t}\right)-\phi(z)+\frac{1}{r}\left\langle z_{t}-z, z-\bar{x}\right\rangle\right\} \\
\leq & t F\left(\lambda_{1} z_{t}+(1-\lambda) b, y\right)+t(1-t)\langle T(z), y-z\rangle+t(1-t) \phi(y)-t(1-t) \phi(z) \\
& \quad+\frac{t(1-t)}{r}\langle y-z, z-\bar{x}\rangle \\
\leq & F\left(\lambda_{1} z_{t}+(1-\lambda) b, y\right)+(1-t)\langle T(z), y-z\rangle+(1-t) \phi(y)-(1-t) \phi(z) \\
& \quad+\frac{(1-t)}{r}\langle y-z, z-\bar{x}\rangle .
\end{aligned}
$$

Letting $t \rightarrow 0$ and therefore $z_{t} \rightarrow z$, and by (A5), we get

$$
F\left(\lambda_{1} z+\left(1-\lambda_{1}\right) b, y\right)+\langle T(z), y-z\rangle+\phi(y)-\phi(z)+\frac{1}{r}\langle y-z, z-\bar{x}\rangle \geq 0
$$

i.e., $z \in J_{r}^{F, T}(\bar{x})$. Hence, from the arbitrariness of $\bar{x}$, we see that $J_{r}^{F, T}(x)$ is nonempty.

(ii) We claim that $J_{r}^{F, T}$ is single-valued. Indeed, for $x \in H_{1}$ and $r>0$, let $z_{1}, z_{2} \in J_{r}^{F, T}(x)$.

Then

$$
F\left(\lambda_{1} z_{1}+\left(1-\lambda_{1}\right) b, z_{2}\right)+\left\langle T\left(z_{1}\right), z_{2}-z_{1}\right\rangle+\phi\left(z_{2}\right)-\phi\left(z_{1}\right)+\frac{1}{r}\left\langle z_{2}-z_{1}, z_{1}-x\right\rangle \geq 0
$$

and

$$
F\left(\lambda_{1} z_{2}+\left(1-\lambda_{1}\right) b, z_{1}\right)+\left\langle T\left(z_{2}\right), z_{1}-z_{2}\right\rangle+\phi\left(z_{1}\right)-\phi\left(z_{2}\right)+\frac{1}{r}\left\langle z_{1}-z_{2}, z_{2}-x\right\rangle \geq 0 .
$$

Adding the above two inequalities, we obtain

$$
\begin{aligned}
& F\left(\lambda_{1} z_{1}+\left(1-\lambda_{1}\right) b, z_{2}\right)+F\left(\lambda_{1} z_{2}+\left(1-\lambda_{1}\right) b, z_{1}\right) \\
& \quad-\left\langle T\left(z_{1}\right)-T\left(z_{2}\right), z_{1}-z_{2}\right\rangle+\frac{1}{r}\left\langle z_{2}-z_{1}, z_{1}-z_{2}\right\rangle \geq 0 .
\end{aligned}
$$

From (A2)-(A3) and $r>0$, we have

$$
\left\langle z_{2}-z_{1}, z_{1}-z_{2}\right\rangle \geq 0,
$$

which implies that $z_{1}=z_{2}$, and hence $J_{r}^{F, T}$ is single-valued. 
(iii) To prove that $J_{r}^{F, T}$ is firmly nonexpansive, for any $x, y \in H_{1}$, we have

$$
\begin{aligned}
& F\left(\lambda_{1} J_{r}^{F, T}(x)+\left(1-\lambda_{1}\right) b, J_{r}^{F, T}(y)\right)+\left\langle T\left(J_{r}^{F, T}(x)\right), J_{r}^{F, T}(y)-J_{r}^{F, T}(x)\right\rangle \\
& \quad+\phi\left(J_{r}^{F, T}(y)\right)-\phi\left(J_{r}^{F, T}(x)\right)+\frac{1}{r}\left\langle J_{r}^{F, T}(y)-J_{r}^{F, T}(x), J_{r}^{F, T}(x)-x\right\rangle \geq 0
\end{aligned}
$$

and

$$
\begin{aligned}
& F\left(\lambda_{1} J_{r}^{F, T}(y)+\left(1-\lambda_{1}\right) b, J_{r}^{F, T}(x)\right)+\left\langle T\left(J_{r}^{F, T}(y)\right), J_{r}^{F, T}(x)-J_{r}^{F, T}(y)\right\rangle \\
& \quad+\phi\left(J_{r}^{F, T}(x)\right)-\phi\left(J_{r}^{F, T}(y)\right)+\frac{1}{r}\left\langle J_{r}^{F, T}(x)-J_{r}^{F, T}(y), J_{r}^{F, T}(y)-y\right\rangle \geq 0 .
\end{aligned}
$$

Adding the above two inequalities, we get

$$
\begin{aligned}
F & \left(\lambda_{1} J_{r}^{F, T}(x)+\left(1-\lambda_{1}\right) b, J_{r}^{F, T}(y)\right)+F\left(\lambda_{1} J_{r}^{F, T}(y)+\left(1-\lambda_{1}\right) b, J_{r}^{F, T}(x)\right) \\
& -\left\langle T\left(J_{r}^{F, T}(y)\right)-T\left(J_{r}^{F, T}(x)\right), J_{r}^{F, T}(y)-J_{r}^{F, T}(x)\right\rangle \\
& +\frac{1}{r}\left\langle J_{r}^{F, T}(y)-J_{r}^{F, T}(x), J_{r}^{F, T}(x)-J_{r}^{F, T}(y)-x+y\right\rangle \geq 0 .
\end{aligned}
$$

Using (A2)-(A3) and $r>0$, we have

$$
\left\langle J_{r}^{F, T}(y)-J_{r}^{F, T}(x), J_{r}^{F, T}(x)-J_{r}^{F, T}(y)-(x-y)\right\rangle \geq 0,
$$

which implies that

$$
\left\|J_{r}^{F, T}(x)-J_{r}^{F, T}(y)\right\|^{2} \leq\left\langle J_{r}^{F, T}(x)-J_{r}^{F, T}(y), x-y\right\rangle .
$$

(iv) Take $x \in C$. Then

$$
\begin{aligned}
x & \in \operatorname{Fix}\left(J_{r}^{F, T}\right) \\
& \Leftrightarrow \quad x=J_{r}^{F, T}(x) \\
& \Leftrightarrow \quad F\left(\lambda_{1} x+\left(1-\lambda_{1}\right) b, y\right)+\langle T(x), y-x\rangle+\phi(y)-\phi(x)+\frac{1}{r}\langle y-x, x-x\rangle \geq 0 \\
& \Leftrightarrow \quad F\left(\lambda_{1} x+\left(1-\lambda_{1}\right) b, y\right)+\langle T(x), y-x\rangle+\phi(y)-\phi(x) \geq 0 \\
& \Leftrightarrow \quad x \in \operatorname{GMEP}(F, T, \phi) .
\end{aligned}
$$

Since $J_{r}^{F, T}$ is firmly nonexpansive, therefore $J_{r}^{F, T}$ is also nonexpansive. By Lemma 2.1, we see that $\operatorname{Fix}\left(J_{r}^{F, T}\right)=\operatorname{GMEP}(F, T, \phi)$ is closed and convex.

Let the mappings $G: Q \times Q \longrightarrow \mathbb{R}$ and $S: Q \longrightarrow Q$ satisfy (A1)-(A7). Let $\varphi: Q \longrightarrow \mathbb{R} \cup$ $\{+\infty\}$ be a proper lower semicontinuous and convex mapping such that $Q \cap \operatorname{dom} \varphi \neq \emptyset$. For $s>0, \lambda_{2} \in(0,1]$, and $u \in H_{2}$, let $J_{s}^{G, S}: H_{2} \longrightarrow Q$ be the resolvent operator of $G$ and $S$, defined by

$$
\begin{aligned}
J_{s}^{G, S}(u)= & \left\{v \in Q: G\left(\lambda_{2} v+\left(1-\lambda_{2}\right) c, w\right)+\langle S(v), w-v\rangle+\varphi(w)-\varphi(v)\right. \\
& \left.+\frac{1}{s}\langle w-v, v-u\rangle \geq 0, \forall w, c \in Q\right\} .
\end{aligned}
$$


Then clearly $J_{s}^{G, S}$ satisfies (i)-(iv) of Lemma 2.2, and $\operatorname{Fix}\left(J_{s}^{G, S}\right)=\operatorname{GMEP}(G, S, \varphi)$.

Lemma 2.3 (Opial's lemma [19]) Let $H$ be a real Hilbert space and $\left\{\mu_{n}\right\}$ be a sequence in $H$ such that there exists a nonempty set $W \subset H$ satisfying the following conditions:

(i) for every $\mu \in W, \lim _{n \rightarrow \infty}\left\|\mu_{n}-\mu\right\|$ exists;

(ii) any weak cluster point of the sequence $\left\{\mu_{n}\right\}$ belongs to $W$.

Then there exists $w^{*} \in W$ such that $\left\{\mu_{n}\right\}$ converges weakly to $w^{*}$.

Lemma 2.4 ([20]) Let $H$ be a real Hilbert space. Then for all $x, y \in H$, we have

$$
\|x-y\|^{2}=\|x\|^{2}-\|y\|^{2}-2\langle x-y, y\rangle .
$$

\section{Convergence results}

In this section, we prove the weak and strong convergence result for split equality generalized mixed equilibrium problem (1.17).

Theorem 3.1 Let $H_{1}, H_{2}$, and $H_{3}$ be real Hilbert spaces, $C \subseteq H_{1}$ and $Q \subseteq H_{2}$ be the nonempty closed convex subsets of $H_{1}$ and $H_{2}$, respectively. Suppose that the bi-mappings $F: C \times C \longrightarrow \mathbb{R}$ and $G: Q \times Q \longrightarrow \mathbb{R}$, and the mappings $T: C \longrightarrow C$ and $S: Q \longrightarrow Q$ satisfy the conditions (A1)-(A7). Let $\phi: C \longrightarrow \mathbb{R} \cup\{+\infty\}$ and $\varphi: Q \rightarrow \mathbb{R} \cup\{+\infty\}$ be the proper lower semicontinuous and convex mappings such that $C \cap \operatorname{dom} \phi \neq \emptyset$ and $Q \cap \operatorname{dom} \varphi \neq \emptyset$. Let $P: H_{1} \longrightarrow H_{1}$ and $Q: H_{2} \longrightarrow H_{2}$ be the two demi-contractive mappings with constants $k_{1}$ and $k_{2}$, respectively, with the condition $k \in(0,1)$, where $k=\max \left\{k_{1}, k_{2}\right\}$ such that $(I-P)$ and $(I-Q)$ are demi-closed at zero, and $\operatorname{Fix}(P) \neq \emptyset$ and $\operatorname{Fix}(Q) \neq \emptyset$. Let $A: H_{1} \longrightarrow H_{3}$ and $B: H_{2} \longrightarrow H_{3}$ be bounded linear mappings. Assume that $\left(x_{1}, y_{1}\right) \in C \times Q$ and the iteration scheme $\left\{\left(x_{n}, y_{n}\right)\right\}$ is defined as follows:

$$
\left\{\begin{array}{l}
F\left(\lambda_{1} u_{n}+\left(1-\lambda_{1}\right) b, u\right)+\left\langle T\left(u_{n}\right), u-u_{n}\right\rangle \\
\quad+\phi(u)-\phi\left(u_{n}\right)+\frac{1}{r_{n}}\left\langle u-u_{n}, u_{n}-x_{n}\right\rangle \geq 0 \\
G\left(\lambda_{2} v_{n}+\left(1-\lambda_{2}\right) c, v\right)+\left\langle S\left(v_{n}\right), v-v_{n}\right\rangle(v) \\
\quad+\varphi-\varphi\left(v_{n}\right)+\frac{1}{r_{n}}\left\langle v-v_{n}, v_{n}-y_{n}\right\rangle \geq 0 \\
x_{n+1}=\left(1-\alpha_{n}\right)\left(u_{n}-\gamma_{n} A^{*}\left(A u_{n}-B v_{n}\right)\right)+\alpha_{n} P\left(u_{n}-\gamma_{n} A^{*}\left(A u_{n}-B v_{n}\right)\right) \\
y_{n+1}=\left(1-\alpha_{n}\right)\left(v_{n}+\gamma_{n} B^{*}\left(A u_{n}-B v_{n}\right)\right)+\alpha_{n} Q\left(v_{n}+\gamma_{n} B^{*}\left(A u_{n}-B v_{n}\right)\right)
\end{array}\right.
$$

for every $u, b \in C, v, c \in Q$, and $n \geq 1$ where $\lambda_{A}$ and $\lambda_{B}$ denote the spectral radii of $A^{*} A$ and $B^{*} B$, respectively, $\left\{\gamma_{n}\right\}$ is a positive real sequence such that $\gamma_{n} \in\left(\epsilon, \frac{2}{\lambda_{A}+\lambda_{B}}-\epsilon\right)$, for sufficiently small $\epsilon,\left\{\alpha_{n}\right\}$ is a sequence in $(k, 1)$, and $\left\{r_{n}\right\} \subset(0, \infty)$ such that the following conditions are satisfied:

(i) for some $\alpha, \beta \in(0,1), 0<\alpha \leq \alpha_{n} \leq \beta<1$;

(ii) $\liminf _{n \rightarrow \infty} r_{n}>0$ and $\lim _{n \rightarrow \infty}\left|r_{n+1}-r_{n}\right|=0$.

If $\Omega:=\operatorname{Fix}(P) \cap \operatorname{Fix}(Q) \cap \operatorname{SEMEP}(F, G, T, S, \phi, \varphi) \neq \emptyset$, then

(I) The sequence $\left\{\left(x_{n}, y_{n}\right)\right\}$ weakly converges to a solution of problem (1.17).

(II) In addition, if $P$ and $Q$ are demi-compact, then $\left\{\left(x_{n}, y_{n}\right)\right\}$ strongly converges to a solution of problem (1.17). 
Proof To prove (I), let $(x, y) \in \Omega$. From Lemma 2.2, we have

$$
\begin{gathered}
\left\|u_{n}-x\right\|=\left\|J_{r_{n}}^{F, T}\left(x_{n}\right)-J_{r_{n}}^{F, T}(x)\right\| \leq\left\|x_{n}-x\right\|, \\
\left\|v_{n}-y\right\|=\left\|J_{r_{n}}^{G, S}\left(y_{n}\right)-J_{r_{n}}^{G, S}(y)\right\| \leq\left\|y_{n}-y\right\| .
\end{gathered}
$$

Since $P$ is a demi-contractive mapping, using the well-known identity (2.1) and Lemma 2.4, we obtain the following estimates:

$$
\begin{aligned}
& \left\|x_{n+1}-x\right\|^{2} \\
& =\left\|\left(1-\alpha_{n}\right)\left(u_{n}-\gamma_{n} A^{*}\left(A u_{n}-B v_{n}\right)\right)+\alpha_{n} P\left(u_{n}-\gamma_{n} A^{*}\left(A u_{n}-B v_{n}\right)\right)-x\right\|^{2} \\
& =\left\|\left(1-\alpha_{n}\right)\left\{\left(u_{n}-\gamma_{n} A^{*}\left(A u_{n}-B v_{n}\right)\right)-x\right\}+\alpha_{n}\left\{P\left(u_{n}-\gamma_{n} A^{*}\left(A u_{n}-B v_{n}\right)\right)-x\right\}\right\|^{2} \\
& =\left(1-\alpha_{n}\right)\left\|\left(u_{n}-\gamma_{n} A^{*}\left(A u_{n}-B v_{n}\right)\right)-x\right\|^{2}+\alpha_{n}\left\|P\left(u_{n}-\gamma_{n} A^{*}\left(A u_{n}-B v_{n}\right)\right)-x\right\|^{2} \\
& -\alpha_{n}\left(1-\alpha_{n}\right)\left\|\left(u_{n}-\gamma_{n} A^{*}\left(A u_{n}-B v_{n}\right)\right)-P\left(u_{n}-\gamma_{n} A^{*}\left(A u_{n}-B v_{n}\right)\right)\right\|^{2} \\
& \leq\left(1-\alpha_{n}\right)\left\|\left(u_{n}-\gamma_{n} A^{*}\left(A u_{n}-B v_{n}\right)\right)-x\right\|^{2}+\alpha_{n}\left\{\left\|\left(u_{n}-\gamma_{n} A^{*}\left(A u_{n}-B v_{n}\right)\right)-x\right\|^{2}\right. \\
& \left.+k_{1}\left\|\left(u_{n}-\gamma_{n} A^{*}\left(A u_{n}-B v_{n}\right)\right)-P\left(u_{n}-\gamma_{n} A^{*}\left(A u_{n}-B v_{n}\right)\right)\right\|^{2}\right\} \\
& -\alpha_{n}\left(1-\alpha_{n}\right)\left\|\left(u_{n}-\gamma_{n} A^{*}\left(A u_{n}-B v_{n}\right)\right)-P\left(u_{n}-\gamma_{n} A^{*}\left(A u_{n}-B v_{n}\right)\right)\right\|^{2} \\
& =\left\|\left(u_{n}-\gamma_{n} A^{*}\left(A u_{n}-B v_{n}\right)\right)-x\right\|^{2} \\
& +k_{1} \alpha_{n}\left\|\left(u_{n}-\gamma_{n} A^{*}\left(A u_{n}-B v_{n}\right)\right)-P\left(u_{n}-\gamma_{n} A^{*}\left(A u_{n}-B v_{n}\right)\right)\right\|^{2} \\
& -\alpha_{n}\left(1-\alpha_{n}\right)\left\|\left(u_{n}-\gamma_{n} A^{*}\left(A u_{n}-B v_{n}\right)\right)-P\left(u_{n}-\gamma_{n} A^{*}\left(A u_{n}-B v_{n}\right)\right)\right\|^{2} \\
& =\left\|\left(u_{n}-\gamma_{n} A^{*}\left(A u_{n}-B v_{n}\right)\right)-x\right\|^{2} \\
& -\alpha_{n}\left(1-k_{1}-\alpha_{n}\right)\left\|\left(u_{n}-\gamma_{n} A^{*}\left(A u_{n}-B v_{n}\right)\right)-P\left(u_{n}-\gamma_{n} A^{*}\left(A u_{n}-B v_{n}\right)\right)\right\|^{2} \\
& =\left\|u_{n}-x\right\|^{2}+\gamma_{n}^{2}\left\|A^{*}\left(A u_{n}-B v_{n}\right)\right\|^{2}-2 \gamma_{n}\left\langle A^{*}\left(A u_{n}-B v_{n}\right), u_{n}-x\right\rangle \\
& -\alpha_{n}\left(1-k_{1}-\alpha_{n}\right)\left\|\left(u_{n}-\gamma_{n} A^{*}\left(A u_{n}-B v_{n}\right)\right)-P\left(u_{n}-\gamma_{n} A^{*}\left(A u_{n}-B v_{n}\right)\right)\right\|^{2} \\
& \leq\left\|x_{n}-x\right\|^{2}+\gamma_{n}^{2}\left\|A^{*}\left(A u_{n}-B v_{n}\right)\right\|^{2}-2 \gamma_{n}\left\langle A^{*}\left(A u_{n}-B v_{n}\right), u_{n}-x\right\rangle \\
& -\alpha_{n}\left(1-k_{1}-\alpha_{n}\right)\left\|\left(u_{n}-\gamma_{n} A^{*}\left(A u_{n}-B v_{n}\right)\right)-P\left(u_{n}-\gamma_{n} A^{*}\left(A u_{n}-B v_{n}\right)\right)\right\|^{2} \text {. }
\end{aligned}
$$

From the definition of the spectral radius $\lambda_{A}$ of $A^{*} A$, we have

$$
\begin{aligned}
\gamma_{n}^{2}\left\|A^{*}\left(A u_{n}-B v_{n}\right)\right\|^{2} & =\gamma_{n}^{2}\left\langle A u_{n}-B v_{n}, A A^{*}\left(A u_{n}-B v_{n}\right)\right\rangle \\
& \leq \lambda_{A} \gamma_{n}^{2}\left\langle A u_{n}-B v_{n}, A u_{n}-B v_{n}\right\rangle \\
& =\lambda_{A} \gamma_{n}^{2}\left\|A u_{n}-B v_{n}\right\|^{2} .
\end{aligned}
$$

Combining (3.4) and (3.5), we have

$$
\begin{aligned}
& \left\|x_{n+1}-x\right\|^{2} \\
& \quad \leq\left\|x_{n}-x\right\|^{2}+\lambda_{A} \gamma_{n}^{2}\left\|A u_{n}-B v_{n}\right\|^{2}-2 \gamma_{n}\left\langle A u_{n}-B v_{n}, A u_{n}-A x\right\rangle \\
& \quad-\alpha_{n}\left(1-k_{1}-\alpha_{n}\right)\left\|\left(u_{n}-\gamma_{n} A^{*}\left(A u_{n}-B v_{n}\right)\right)-P\left(u_{n}-\gamma_{n} A^{*}\left(A u_{n}-B v_{n}\right)\right)\right\|^{2} .
\end{aligned}
$$


Similarly, the last equality of the iterative scheme (3.1) leads to

$$
\begin{aligned}
& \left\|y_{n+1}-y\right\|^{2} \\
& \quad \leq\left\|y_{n}-y\right\|^{2}+\lambda_{B} \gamma_{n}^{2}\left\|A u_{n}-B v_{n}\right\|^{2}+2 \gamma_{n}\left\langle A u_{n}-B v_{n}, B v_{n}-B y\right\rangle \\
& \quad-\alpha_{n}\left(1-k_{2}-\alpha_{n}\right)\left\|\left(v_{n}+\gamma_{n} B^{*}\left(A u_{n}-B v_{n}\right)\right)-Q\left(v_{n}+\gamma_{n} B^{*}\left(A u_{n}-B v_{n}\right)\right)\right\|^{2} .
\end{aligned}
$$

Adding the inequalities (3.6) and (3.7), using $k=\max \left\{k_{1}, k_{2}\right\}$ and $A x=B y$, we get

$$
\begin{aligned}
\| x_{n+1} & -x\left\|^{2}+\right\| y_{n+1}-y \|^{2} \\
\leq & \left\|x_{n}-x\right\|^{2}+\left\|y_{n}-y\right\|^{2}+\left(\lambda_{A} \gamma_{n}^{2}+\lambda_{B} \gamma_{n}^{2}\right)\left\|A u_{n}-B v_{n}\right\|^{2}-2 \gamma_{n}\left\|A u_{n}-B v_{n}\right\|^{2} \\
& \quad-\alpha_{n}\left(1-k-\alpha_{n}\right)\left\{\left\|\left(u_{n}-\gamma_{n} A^{*}\left(A u_{n}-B v_{n}\right)\right)-P\left(u_{n}-\gamma_{n} A^{*}\left(A u_{n}-B v_{n}\right)\right)\right\|^{2}\right. \\
& \left.+\left\|\left(v_{n}+\gamma_{n} B^{*}\left(A u_{n}-B v_{n}\right)\right)-Q\left(v_{n}+\gamma_{n} B^{*}\left(A u_{n}-B v_{n}\right)\right)\right\|^{2}\right\} \\
= & \left\|x_{n}-x\right\|^{2}+\left\|y_{n}-y\right\|^{2}-\gamma_{n}\left(2-\gamma_{n}\left(\lambda_{A}+\lambda_{B}\right)\right)\left\|A u_{n}-B v_{n}\right\|^{2} \\
& -\alpha_{n}\left(1-k-\alpha_{n}\right)\left\{\left\|\left(u_{n}-\gamma_{n} A^{*}\left(A u_{n}-B v_{n}\right)\right)-P\left(u_{n}-\gamma_{n} A^{*}\left(A u_{n}-B v_{n}\right)\right)\right\|^{2}\right. \\
& \left.+\left\|\left(v_{n}+\gamma_{n} B^{*}\left(A u_{n}-B v_{n}\right)\right)-Q\left(v_{n}+\gamma_{n} B^{*}\left(A u_{n}-B v_{n}\right)\right)\right\|^{2}\right\} .
\end{aligned}
$$

Now, put $\Omega_{n}(x, y)=\left\|x_{n}-x\right\|^{2}+\left\|y_{n}-y\right\|^{2}$. Therefore from (3.8), we have

$$
\begin{aligned}
\Omega_{n+1}(x, y) \leq & \Omega_{n}(x, y)-\gamma_{n}\left(2-\gamma_{n}\left(\lambda_{A}+\lambda_{B}\right)\right)\left\|A u_{n}-B v_{n}\right\|^{2} \\
& -\alpha_{n}\left(1-k-\alpha_{n}\right)\left\{\left\|\left(u_{n}-\gamma_{n} A^{*}\left(A u_{n}-B v_{n}\right)\right)-P\left(u_{n}-\gamma_{n} A^{*}\left(A u_{n}-B v_{n}\right)\right)\right\|^{2}\right. \\
& \left.+\left\|\left(v_{n}+\gamma_{n} B^{*}\left(A u_{n}-B v_{n}\right)\right)-Q\left(v_{n}+\gamma_{n} B^{*}\left(A u_{n}-B v_{n}\right)\right)\right\|^{2}\right\}
\end{aligned}
$$

As $\alpha_{n} \in(k, 1)$ and $\gamma_{n} \in\left(\epsilon, \frac{2}{\lambda_{A}+\lambda_{B}}-\epsilon\right)$, we have $2-\gamma_{n}\left(\lambda_{A}+\lambda_{B}\right)>0$ and $\left(1-k-\alpha_{n}\right)>0$. It follows from (3.9) that

$$
\Omega_{n+1}(x, y) \leq \Omega_{n}(x, y)
$$

Hence, the sequence $\left\{\Omega_{n}(x, y)\right\}$ is non-increasing and lower bounded by 0 . Therefore, it converges to some finite limit, say $\sigma(x, y)$. So, condition (i) of Lemma 2.3 is satisfied with $\mu_{n}=\left(x_{n}, y_{n}\right), \mu^{*}=(x, y)$, and $W=\Omega$. It follows from inequality (3.9) and the convergence of the sequence $\left\{\Omega_{n}(x, y)\right\}$ that

$$
\begin{aligned}
& \lim _{n \rightarrow \infty}\left\|A u_{n}-B v_{n}\right\|=0, \\
& \lim _{n \rightarrow \infty}\left\|\left(u_{n}-\gamma_{n} A^{*}\left(A u_{n}-B v_{n}\right)\right)-P\left(u_{n}-\gamma_{n} A^{*}\left(A u_{n}-B v_{n}\right)\right)\right\|=0,
\end{aligned}
$$

and

$$
\lim _{n \rightarrow \infty}\left\|\left(v_{n}+\gamma_{n} B^{*}\left(A u_{n}-B v_{n}\right)\right)-Q\left(v_{n}+\gamma_{n} B^{*}\left(A u_{n}-B v_{n}\right)\right)\right\|=0 .
$$

Moreover, as $\left\{\Omega_{n}(x, y)\right\}$ converges to a finite limit and $\left\|x_{n}-x\right\|^{2} \leq \Omega_{n}(x, y), \| y_{n}-$ $y \|^{2} \leq \Omega_{n}(x, y)$, we see that $\left\{x_{n}\right\}$ and $\left\{y_{n}\right\}$ are bounded and $\limsup _{n \rightarrow \infty}\left\|x_{n}-x\right\|$ and 
$\lim \sup _{n \rightarrow \infty}\left\|y_{n}-y\right\|$ exist. From (3.2) and (3.3), we get $\limsup _{n \rightarrow \infty}\left\|u_{n}-x\right\|$ and $\limsup _{n \rightarrow \infty}\left\|v_{n}-y\right\|$ also exist. Let $x^{*}$ and $y^{*}$ be weak limit points of the sequences $\left\{x_{n}\right\}$ and $\left\{y_{n}\right\}$, respectively. Also, $\left\{u_{n}-\gamma_{n} A^{*}\left(A u_{n}-B v_{n}\right)\right\}$ weakly converges to $x^{*}$ and $\left\{v_{n}+\gamma_{n} B^{*}\left(A u_{n}-B v_{n}\right)\right\}$ weakly converges to $y^{*}$. Using Lemma 2.4 , we have

$$
\begin{aligned}
\left\|x_{n+1}-x_{n}\right\|^{2}= & \left\|x_{n+1}-x-x_{n}+x\right\|^{2} \\
= & \left\|x_{n+1}-x\right\|^{2}-\left\|x_{n}-x\right\|^{2}-2\left\langle x_{n+1}-x_{n}, x_{n}-x\right\rangle \\
= & \left\|x_{n+1}-x\right\|^{2}-\left\|x_{n}-x\right\|^{2}-2\left\langle x_{n+1}-x^{*}, x_{n}-x\right\rangle \\
& +2\left\langle x_{n}-x^{*}, x_{n}-x\right\rangle .
\end{aligned}
$$

Therefore,

$$
\limsup _{n \rightarrow \infty}\left\|x_{n+1}-x_{n}\right\|=0
$$

Similarly, we obtain

$$
\limsup _{n \rightarrow \infty}\left\|y_{n+1}-y_{n}\right\|=0
$$

We conclude that

$$
\lim _{n \rightarrow \infty}\left\|x_{n+1}-x_{n}\right\|=0
$$

and

$$
\lim _{n \rightarrow \infty}\left\|y_{n+1}-y_{n}\right\|=0
$$

From Lemma 2.2, we have $u_{n}=J_{r_{n}}^{F, T}\left(x_{n}\right)$ and $u_{n+1}=J_{r_{n+1}}^{F, T}\left(x_{n+1}\right)$. Therefore, for all $u \in C$, we have

$$
\begin{aligned}
& F\left(\lambda_{1} u_{n}+\left(1-\lambda_{1}\right) b, u\right)+\left\langle T\left(u_{n}\right), u-u_{n}\right\rangle \\
& \quad+\phi(u)-\phi\left(u_{n}\right)+\frac{1}{r_{n}}\left\langle u-u_{n}, u_{n}-x_{n}\right\rangle \geq 0
\end{aligned}
$$

and

$$
\begin{aligned}
& F\left(\lambda_{1} u_{n+1}+\left(1-\lambda_{1}\right) b, u\right)+\left\langle T\left(u_{n+1}\right), u-u_{n+1}\right\rangle \\
& \quad+\phi(u)-\phi\left(u_{n+1}\right)+\frac{1}{r_{n+1}}\left\langle u-u_{n+1}, u_{n+1}-x_{n+1}\right\rangle \geq 0 .
\end{aligned}
$$

Putting $u=u_{n}$ in (3.16) and $u=u_{n+1}$ in (3.15), and adding together the resulting inequalities, we have

$$
\begin{aligned}
0 \leq & F\left(\lambda_{1} u_{n+1}+\left(1-\lambda_{1}\right) b, u_{n}\right)+F\left(\lambda_{1} u_{n}+\left(1-\lambda_{1}\right) b, u_{n+1}\right)+\left\langle T\left(u_{n+1}\right), u_{n}-u_{n+1}\right\rangle \\
& +\left\langle T\left(u_{n}\right), u_{n+1}-u_{n}\right\rangle+\frac{1}{r_{n+1}}\left\langle u_{n}-u_{n+1}, u_{n+1}-x_{n+1}\right\rangle+\frac{1}{r_{n}}\left\langle u_{n+1}-u_{n}, u_{n}-x_{n}\right\rangle .
\end{aligned}
$$


By using (A2)-(A3), we have

$$
\begin{aligned}
0 & \leq \frac{1}{r_{n+1}}\left\langle u_{n}-u_{n+1}, u_{n+1}-x_{n+1}\right\rangle+\frac{1}{r_{n}}\left\langle u_{n+1}-u_{n}, u_{n}-x_{n}\right\rangle \\
& \leq\left\langle u_{n+1}-u_{n}, \frac{u_{n}-x_{n}}{r_{n}}-\frac{u_{n+1}-x_{n+1}}{r_{n+1}}\right\rangle \\
& =\left\langle u_{n+1}-u_{n}, u_{n}-x_{n}-\frac{r_{n}}{r_{n+1}}\left(u_{n+1}-x_{n+1}\right)\right\rangle \\
& =\left\langle u_{n+1}-u_{n}, u_{n}-u_{n+1}+u_{n+1}-x_{n}-\frac{r_{n}}{r_{n+1}}\left(u_{n+1}-x_{n+1}\right)\right\rangle \\
& =\left\langle u_{n+1}-u_{n}, u_{n}-u_{n+1}\right\rangle+\left\langle u_{n+1}-u_{n}, x_{n+1}-x_{n}+\left(1-\frac{r_{n}}{r_{n+1}}\right)\left(u_{n+1}-x_{n+1}\right)\right\rangle \\
& =-\left\|u_{n+1}-u_{n}\right\|^{2}+\left\langle u_{n+1}-u_{n}, x_{n+1}-x_{n}+\left(1-\frac{r_{n}}{r_{n+1}}\right)\left(u_{n+1}-x_{n+1}\right)\right\rangle
\end{aligned}
$$

which implies that

$$
\left\|u_{n+1}-u_{n}\right\|^{2} \leq\left\|u_{n+1}-u_{n}\right\|\left\{\left\|x_{n+1}-x_{n}\right\|+\left|1-\frac{r_{n}}{r_{n+1}}\right|\left\|u_{n+1}-x_{n+1}\right\|\right\} .
$$

Thus,

$$
\left\|u_{n+1}-u_{n}\right\| \leq\left\|x_{n+1}-x_{n}\right\|+\left|1-\frac{r_{n}}{r_{n+1}}\right|\left\|u_{n+1}-x_{n+1}\right\| .
$$

Using (3.13) and condition (ii) of the hypothesis, (3.17) implies that

$$
\lim _{n \rightarrow \infty}\left\|u_{n+1}-u_{n}\right\|=0
$$

Similarly, using the same arguments as above, we have

$$
\lim _{n \rightarrow \infty}\left\|v_{n+1}-v_{n}\right\|=0 .
$$

From (3.6) and (3.7), we have

$$
\begin{aligned}
& \left\|x_{n+1}-x\right\|^{2} \\
& \quad \leq\left\|u_{n}-x\right\|^{2}+\lambda_{A} \gamma_{n}^{2}\left\|A u_{n}-B v_{n}\right\|^{2}-2 \gamma_{n}\left\langle A u_{n}-B v_{n}, A u_{n}-A x\right\rangle \\
& \quad-\alpha_{n}\left(1-k_{1}-\alpha_{n}\right)\left\|\left(u_{n}-\gamma_{n} A^{*}\left(A u_{n}-B v_{n}\right)\right)-P\left(u_{n}-\gamma_{n} A^{*}\left(A u_{n}-B v_{n}\right)\right)\right\|^{2}
\end{aligned}
$$

and

$$
\begin{aligned}
& \left\|y_{n+1}-y\right\|^{2} \\
& \quad \leq\left\|v_{n}-y\right\|^{2}+\lambda_{B} \gamma_{n}^{2}\left\|A u_{n}-B v_{n}\right\|^{2}+2 \gamma_{n}\left\langle A u_{n}-B v_{n}, B v_{n}-B y\right\rangle \\
& \quad-\alpha_{n}\left(1-k_{2}-\alpha_{n}\right)\left\|\left(v_{n}+\gamma_{n} B^{*}\left(A u_{n}-B v_{n}\right)\right)-Q\left(v_{n}+\gamma_{n} B^{*}\left(A u_{n}-B v_{n}\right)\right)\right\|^{2} .
\end{aligned}
$$


Adding the inequalities (3.20) and (3.21), using $k=\max \left\{k_{1}, k_{2}\right\}$ and $A x=B y$, we obtain

$$
\begin{aligned}
\| x_{n+1} & -x\left\|^{2}+\right\| y_{n+1}-y \|^{2} \\
\leq & \left\|u_{n}-x\right\|^{2}+\left\|v_{n}-y\right\|^{2}-\gamma_{n}\left(2-\gamma_{n}\left(\lambda_{A}+\lambda_{B}\right)\right)\left\|A u_{n}-B v_{n}\right\|^{2} \\
& \quad-\alpha_{n}\left(1-k-\alpha_{n}\right)\left\{\left\|\left(u_{n}-\gamma_{n} A^{*}\left(A u_{n}-B v_{n}\right)\right)-P\left(u_{n}-\gamma_{n} A^{*}\left(A u_{n}-B v_{n}\right)\right)\right\|^{2}\right. \\
& \left.+\left\|\left(v_{n}+\gamma_{n} B^{*}\left(A u_{n}-B v_{n}\right)\right)-Q\left(v_{n}+\gamma_{n} B^{*}\left(A u_{n}-B v_{n}\right)\right)\right\|^{2}\right\},
\end{aligned}
$$

where

$$
\begin{aligned}
\left\|u_{n}-x\right\|^{2}=\left\|J_{r_{n}}^{F, T}\left(x_{n}\right)-J_{r_{n}}^{F, T}(x)\right\|^{2} & \leq\left\langle x_{n}-x, u_{n}-x\right\rangle \\
& =\frac{1}{2}\left\{\left\|x_{n}-x\right\|^{2}+\left\|u_{n}-x\right\|^{2}-\left\|x_{n}-u_{n}\right\|^{2}\right\}
\end{aligned}
$$

and

$$
\begin{aligned}
\left\|v_{n}-y\right\|^{2}=\left\|J_{r_{n}}^{G, S}\left(y_{n}\right)-J_{r_{n}}^{G, S}(y)\right\|^{2} & \leq\left\langle y_{n}-y, v_{n}-y\right\rangle \\
& =\frac{1}{2}\left\{\left\|y_{n}-y\right\|^{2}+\left\|v_{n}-y\right\|^{2}-\left\|y_{n}-v_{n}\right\|^{2}\right\} .
\end{aligned}
$$

From (3.22)-(3.24), we conclude that

$$
\begin{aligned}
\| x_{n}- & u_{n}\left\|^{2}+\right\| y_{n}-v_{n} \|^{2} \\
\leq & \left\|x_{n}-x\right\|^{2}-\left\|x_{n+1}-x\right\|^{2}+\left\|y_{n}-y\right\|^{2}-\left\|y_{n+1}-y\right\|^{2} \\
& -\gamma_{n}\left(2-\gamma_{n}\left(\lambda_{A}+\lambda_{B}\right)\right)\left\|A u_{n}-B v_{n}\right\|^{2} \\
& -\alpha_{n}\left(1-k-\alpha_{n}\right)\left\{\left\|\left(u_{n}-\gamma_{n} A^{*}\left(A u_{n}-B v_{n}\right)\right)-P\left(u_{n}-\gamma_{n} A^{*}\left(A u_{n}-B v_{n}\right)\right)\right\|^{2}\right. \\
& \left.+\left\|\left(v_{n}+\gamma_{n} B^{*}\left(A u_{n}-B v_{n}\right)\right)-Q\left(v_{n}+\gamma_{n} B^{*}\left(A u_{n}-B v_{n}\right)\right)\right\|^{2}\right\} .
\end{aligned}
$$

By using (3.10)-(3.14), we have

$$
\begin{aligned}
& \lim _{n \rightarrow \infty}\left\|x_{n}-u_{n}\right\|=0, \\
& \lim _{n \rightarrow \infty}\left\|y_{n}-v_{n}\right\|=0 .
\end{aligned}
$$

Hence, $u_{n} \rightarrow x^{*}$ and $v_{n} \rightarrow y^{*}$, respectively.

Since $P$ is $k_{1}$-demi-contractive mapping and $(I-P)$ is demi-closed at 0 , we have

$$
\begin{aligned}
&\left\|u_{n}-P u_{n}\right\| \\
&=\left\|u_{n}-x_{n+1}+x_{n} n+1-P u_{n}\right\| \\
& \leq\left\|u_{n}-x_{n+1}\right\|+\left\|x_{n} n+1-P u_{n}\right\| \\
&=\left\|u_{n}-u_{n+1}+u_{n+1}-x_{n+1}\right\| \\
&+\left\|\left(1-\alpha_{n}\right)\left(u_{n}-\gamma_{n} A^{*}\left(A u_{n}-B v_{n}\right)\right)+\alpha_{n} P\left(u_{n}-\gamma_{n} A^{*}\left(A u_{n}-B v_{n}\right)\right)-P u_{n}\right\| \\
& \leq\left\|u_{n}-u_{n+1}\right\|+\left\|u_{n+1}-x_{n+1}\right\|+\left\|\left(u_{n}-\gamma_{n} A^{*}\left(A u_{n}-B v_{n}\right)\right)-P u_{n}\right\|
\end{aligned}
$$




$$
\begin{aligned}
& +\alpha_{n}\left\|\left(u_{n}-\gamma_{n} A^{*}\left(A u_{n}-B v_{n}\right)\right)-P\left(u_{n}-\gamma_{n} A^{*}\left(A u_{n}-B v_{n}\right)\right)\right\| \\
\leq & \left\|u_{n}-u_{n+1}\right\|+\left\|u_{n+1}-x_{n+1}\right\|+\frac{1+\sqrt{k}}{1-\sqrt{k}}\left\{\left|\gamma_{n}\right|\left\|A^{*}\right\|\left\|A u_{n}-B v_{n}\right\|\right\} \\
& +\alpha_{n}\left\|\left(u_{n}-\gamma_{n} A^{*}\left(A u_{n}-B v_{n}\right)\right)-P\left(u_{n}-\gamma_{n} A^{*}\left(A u_{n}-B v_{n}\right)\right)\right\| .
\end{aligned}
$$

Using (3.10), (3.11), (3.18), and (3.26), we have

$$
\lim _{n \rightarrow \infty}\left\|u_{n}-P u_{n}\right\|=0
$$

Similarly, using the same steps as above for $Q$, we have

$$
\lim _{n \rightarrow \infty}\left\|v_{n}-Q v_{n}\right\|=0
$$

Since

$$
\begin{aligned}
\left\|x_{n}-P x_{n}\right\| & =\left\|x_{n}-u_{n}+u_{n}-P u_{n}+P u_{n}-P x_{n}\right\| \\
& \leq\left\|x_{n}-u_{n}\right\|+\left\|u_{n}-P u_{n}\right\|+\left\|P u_{n}-P x_{n}\right\| \\
& \leq\left\|x_{n}-u_{n}\right\|+\left\|u_{n}-P u_{n}\right\|+\frac{1+\sqrt{k}}{1-\sqrt{k}}\left\|u_{n}-x_{n}\right\| \\
& =\frac{2}{1-\sqrt{k}}\left\|x_{n}-u_{n}\right\|+\left\|u_{n}-P u_{n}\right\|,
\end{aligned}
$$

it follows from (3.26) and (3.28) that

$$
\lim _{n \rightarrow \infty}\left\|x_{n}-P x_{n}\right\|=0
$$

Similarly, we have

$$
\lim _{n \rightarrow \infty}\left\|y_{n}-Q y_{n}\right\|=0
$$

As $\left\{x_{n}\right\}$ and $\left\{y_{n}\right\}$ weakly converge to $x^{*}$ and $y^{*}$, respectively, and $(I-P)$ and $(I-Q)$ are demi-closed at 0 , it follows from (3.30) and (3.31) that $x^{*} \in \operatorname{Fix}(P)$ and $y^{*} \in \operatorname{Fix}(Q)$. Every Hilbert space satisfies Opial's condition, which shows that the weakly subsequential limit of $\left\{\left(x_{n}, y_{n}\right)\right\}$ is unique.

Now, we show that $x^{*} \in \operatorname{GMEP}(F, T, \phi)$ and $y^{*} \in \operatorname{GMEP}(G, S, \varphi)$. Since $u_{n}=J_{r_{n}}^{F, T}\left(x_{n}\right)$, we have, for all $b, u \in C$ and $\lambda \in(0,1]$,

$$
F\left(\lambda_{1} u_{n}+\left(1-\lambda_{1}\right) b, u\right)+\left\langle T\left(u_{n}\right), u-u_{n}\right\rangle+\phi(u)-\phi\left(u_{n}\right)+\frac{1}{r_{n}}\left\langle u-u_{n}, u_{n}-x_{n}\right\rangle \geq 0 .
$$

Using (A2) and (A3), we get

$$
\begin{aligned}
\phi(u)-\phi\left(u_{n}\right)+\frac{1}{r_{n}}\left\langle u-u_{n}, u_{n}-x_{n}\right\rangle & \geq-F\left(\lambda_{1} u_{n}+\left(1-\lambda_{1}\right) b, u\right)-\left\langle T\left(u_{n}\right), u-u_{n}\right\rangle \\
& \geq F\left(\lambda_{1} u+\left(1-\lambda_{1}\right) b, u_{n}\right)+\left\langle T(u), u_{n}-u\right\rangle,
\end{aligned}
$$


and hence

$$
\phi(u)-\phi\left(u_{n_{k}}\right)+\frac{1}{r_{n_{k}}}\left\langle u-u_{n_{k}}, u_{n_{k}}-x_{n_{k}}\right\rangle \geq F\left(\lambda_{1} u+\left(1-\lambda_{1}\right) b, u_{n_{k}}\right)+\left\langle T(u), u_{n_{k}}-u\right\rangle .
$$

From (3.26), we have $u_{n_{k}} \rightarrow x^{*}$. It shows that $\lim _{k \rightarrow \infty} \frac{\left\|u_{n_{k}}-x_{n_{k}}\right\|}{r_{n_{k}}}=0$, and from the lower semicontinuity of $\phi$, we have

$$
F\left(\lambda_{1} u+\left(1-\lambda_{1}\right) b, x^{*}\right)+\left\langle T(u), x^{*}-u\right\rangle+\phi\left(x^{*}\right)-\phi(u) \leq 0, \quad \forall b, u \in C .
$$

Set $u_{t}=t u+(1-t) x^{*}$, for all $t \in(0,1]$ and $u \in C$. Since $C$ is a convex set, $u_{t} \in C$. Hence from (3.32), we have

$$
F\left(\lambda_{1} u_{t}+\left(1-\lambda_{1}\right) b, x^{*}\right)+\left\langle T\left(u_{t}\right), x^{*}-u_{t}\right\rangle+\phi\left(x^{*}\right)-\phi\left(u_{t}\right) \leq 0 .
$$

Using the conditions (A1)-(A4), convexity of $\phi$, and (3.33), we get

$$
\begin{aligned}
0= & F\left(\lambda_{1} u_{t}+\left(1-\lambda_{1}\right) b, u_{t}\right)+(1-t)\left\langle T\left(u_{t}\right), u_{t}-u_{t}\right\rangle+\phi\left(u_{t}\right)-\phi\left(u_{t}\right) \\
\leq & t F\left(\lambda_{1} u_{t}+\left(1-\lambda_{1}\right) b, u\right)+(1-t) F\left(\lambda_{1} u_{t}+\left(1-\lambda_{1}\right) b, x^{*}\right)+t \phi(u)+(1-t) \phi\left(x^{*}\right) \\
& -\phi\left(u_{t}\right)+(1-t)\left\langle T\left(u_{t}\right), u_{t}-x^{*}\right\rangle+(1-t)\left\langle T\left(u_{t}\right), x^{*}-u_{t}\right\rangle \\
= & t\left\{F\left(\lambda_{1} u_{t}+\left(1-\lambda_{1}\right) b, u\right)+(1-t)\left\langle T\left(u_{t}\right), u-x^{*}\right\rangle+\phi(u)-\phi\left(u_{t}\right)\right\} \\
& \times(1-t)\left\{F\left(\lambda_{1} u_{t}+\left(1-\lambda_{1}\right) b, x^{*}\right)+\left\langle T\left(u_{t}\right), x^{*}-u_{t}\right\rangle+\phi\left(x^{*}\right)-\phi\left(u_{t}\right)\right\} \\
\leq & t\left\{F\left(\lambda_{1} u_{t}+\left(1-\lambda_{1}\right) b, u\right)+(1-t)\left\langle T\left(u_{t}\right), u-x^{*}\right\rangle+\phi(u)-\phi\left(u_{t}\right)\right\},
\end{aligned}
$$

which implies that

$$
F\left(\lambda_{1} u_{t}+\left(1-\lambda_{1}\right) b, u\right)+(1-t)\left\langle T\left(u_{t}\right), u-x^{*}\right\rangle+\phi(u)-\phi\left(u_{t}\right) \geq 0, \quad \forall u, b \in C .
$$

Let $t \rightarrow 0$ and therefore $u_{t} \rightarrow x^{*}$. Using the conditions (A5)-(A6) and proper lower semicontinuity of $\phi$, we have

$$
F\left(\lambda_{1} x^{*}+\left(1-\lambda_{1}\right) b, u\right)+\left\langle T\left(x^{*}\right), u-x^{*}\right\rangle+\phi(u)-\phi\left(x^{*}\right) \geq 0, \quad \forall u, b \in C,
$$

which shows that $x^{*} \in \operatorname{GMEP}(F, T, \phi)$. Using the equivalent assertions to the above, we obtain $y^{*} \in \operatorname{GMEP}(G, S, \varphi)$.

Since $A: H_{1} \longrightarrow H_{3}$ and $B: H_{2} \longrightarrow H_{3}$ are bounded linear mappings, and $\left\{u_{n}\right\}$ and $\left\{v_{n}\right\}$ converges weakly to $x^{*}$ and $y^{*}$, respectively, for arbitrary $f \in H_{3}^{*}$, we have

$$
f\left(A u_{n}\right)=(f \circ A)\left(u_{n}\right) \quad \longrightarrow \quad(f \circ A)\left(x^{*}\right)=f\left(A x^{*}\right) .
$$

Likewise,

$$
f\left(B v_{n}\right)=(f \circ B)\left(v_{n}\right) \quad \longrightarrow \quad(f \circ B)\left(y^{*}\right)=f\left(B y^{*}\right) .
$$


Therefore, we have

$$
A u_{n}-B v_{n} \rightarrow A x^{*}-B y^{*},
$$

which implies that

$$
\left\|A x^{*}-B y^{*}\right\| \leq \liminf _{n \rightarrow \infty}\left\|A u_{n}-B v_{n}\right\|=0,
$$

so that $A x^{*}=B y^{*}$. This implies that $\left(x^{*}, y^{*}\right) \in \operatorname{SEMEP}(F, G, T, S, \phi, \varphi)$. Therefore, $\left(x^{*}, y^{*}\right) \in \Omega$.

Finally, we conclude that

1. for each $\left(x^{*}, y^{*}\right) \in \Omega, \lim _{n \rightarrow \infty}\left(\left\|x_{n}-x^{*}\right\|+\left\|y_{n}-y^{*}\right\|\right)$ exists;

2. each weak cluster point of the sequence $\left\|\left(x^{*}, y^{*}\right)\right\|$ belongs to $\Omega$.

On taking $H=H_{1} \times H_{2}$ with the norm $\|(x, y)\|=\sqrt{\left(\|x\|^{2}+\|y\|^{2}\right)}, W=\Omega, \mu_{n}=\left(x_{n}, y_{n}\right)$, and $\mu=\left(x^{*}, y^{*}\right)$ in Lemma 2.3 , we see that there exists $(\bar{x}, \bar{y}) \in \Omega$ such that $x_{n}-\bar{x}$ and $y_{n} \rightarrow \bar{y}$. Therefore, the sequence $\left\{\left(x_{n}, y_{n}\right)\right\}$ generated by the iterative scheme (3.1) weakly converges to a solution of problem (1.17) in $\Omega$. This completes the proof of conjecture (I).

We now prove the strong convergence conjecture (II).

Since $P$ and $Q$ are demi-compact, $\left\{x_{n}\right\}$ and $\left\{y_{n}\right\}$ are bounded, and $\lim _{n \rightarrow \infty}\left\|x_{n}-P x_{n}\right\|=0$, $\lim _{n \rightarrow \infty}\left\|y_{n}-Q y_{n}\right\|=0$, there exist (without loss of generality) subsequences $\left\{x_{n_{k}}\right\}$ of $\left\{x_{n}\right\}$ and $\left\{y_{n_{k}}\right\}$ of $\left\{y_{n}\right\}$ such that $\left\{x_{n_{k}}\right\}$ and $\left\{y_{n_{k}}\right\}$ converge strongly to some points $u^{*}$ and $v^{*}$, respectively. Since $\left\{x_{n_{k}}\right\}$ and $\left\{y_{n_{k}}\right\}$ converge weakly to $x^{*}$ and $y^{*}$, respectively, this implies that $x^{*}=u^{*}$ and $y^{*}=v^{*}$. It follows from the demi-closedness of $P$ and $Q$ that $x^{*} \in \operatorname{Fix}(P)$ and $y^{*} \in \operatorname{Fix}(Q)$. Using similar steps to the previous ones, we get $x^{*} \in \operatorname{GMEP}(F, T, \phi)$ and $y^{*} \in \operatorname{GMEP}(G, S, \varphi)$. Thus, we have

$$
\left\|A x^{*}-B y^{*}\right\|=\lim _{k \rightarrow \infty}\left\|A x_{n_{k}}-B y_{n_{k}}\right\|=0 .
$$

This implies that $A x^{*}=B y^{*}$. Hence $\left(x^{*}, y^{*}\right) \in \Omega$. On the other hand, since $\Omega_{n}(x, y)=\| x_{n}-$ $x\left\|^{2}+\right\| y_{n}-y \|^{2}$, for any $(x, y) \in \Omega$, we know that $\lim _{k \rightarrow \infty} \Omega_{n}\left(x^{*}, y^{*}\right)=0$. From conjecture (I), we see that $\lim _{n \rightarrow \infty} \Omega_{n}\left(x^{*}, y^{*}\right)$ exists, therefore $\lim _{n \rightarrow \infty} \Omega_{n}\left(x^{*}, y^{*}\right)=0$. So, the iterative scheme (3.1) converges strongly to a solution of problem (1.17). This completes the proof of the conjecture (II).

Remark 3.1 The convergence theorem, Theorem 3.1, generalizes, extends, and unifies some well-known weak and strong convergence results of Moudafi et al. [12], Ma et al. [15], Ma et al. [13] and Chidume et al. [16] as we considered the class of demi-contractive mappings, which is much larger than the class of nonexpansive mappings, firmly quasinonexpansive mappings. Also, we studied the split equality generalized equilibrium problem (1.17), which is a more general problem than the split equality problem (1.9), the split equality mixed equilibrium problem (1.15), etc.

On taking $F=G=0, T=S=0$, and $\phi=\varphi=0$ in Theorem 3.1, we get the following convergence theorem for the split equality problem (1.9).

Corollary 3.1 Let $H_{1}, H_{2}$, and $H_{3}$ be real Hilbert spaces, $C \subseteq H_{1}$ and $Q \subseteq H_{2}$ be the nonempty closed convex subsets of $H_{1}$ and $H_{2}$, respectively. Let $P: H_{1} \longrightarrow H_{1}$ and $Q: H_{2} \longrightarrow$ 
$\mathrm{H}_{2}$ be two demi-contractive mappings with constants $k_{1}$ and $k_{2}$, respectively, with the condition $k \in(0,1)$, where $k=\max \left\{k_{1}, k_{2}\right\}$ such that $(I-P)$ and $(I-Q)$ are demi-closed at zero, and $\operatorname{Fix}(P) \neq \emptyset$ and $\operatorname{Fix}(Q) \neq \emptyset$. Let $A: H_{1} \longrightarrow H_{3}$ and $B: H_{2} \longrightarrow H_{3}$ be bounded linear mappings. Assume that $\left(x_{1}, y_{1}\right) \in C \times Q$ and the iteration scheme $\left\{\left(x_{n}, y_{n}\right)\right\}$ is defined as follows:

$$
\left\{\begin{array}{l}
x_{n+1}=\left(1-\alpha_{n}\right)\left(x_{n}-\gamma_{n} A^{*}\left(A x_{n}-B y_{n}\right)\right)+\alpha_{n} P\left(x_{n}-\gamma_{n} A^{*}\left(A x_{n}-B y_{n}\right)\right) \\
y_{n+1}=\left(1-\alpha_{n}\right)\left(y_{n}+\gamma_{n} B^{*}\left(A x_{n}-B y_{n}\right)\right)+\alpha_{n} Q\left(y_{n}+\gamma_{n} B^{*}\left(A x_{n}-B y_{n}\right)\right),
\end{array}\right.
$$

where $\lambda_{A}$ and $\lambda_{B}$ denote the spectral radii of $A^{*} A$ and $B^{*} B$, respectively, $\left\{\gamma_{n}\right\}$ is a positive real sequence such that $\gamma_{n} \in\left(\epsilon, \frac{2}{\lambda_{A}+\lambda_{B}}-\epsilon\right)$ (for $\epsilon$ small enough), $\left\{\alpha_{n}\right\}$ is a sequence in $(k, 1)$ such that for some $\alpha, \beta \in(0,1), 0<\alpha \leq \alpha_{n} \leq \beta<1$.

If $\Omega:=\operatorname{Fix}(P) \cap \operatorname{Fix}(Q) \cap S E P \neq \emptyset$, then:

(I) The sequence $\left\{\left(x_{n}, y_{n}\right)\right\}$ weakly converges to a solution of problem (1.9).

(II) In addition, if $P$ and $Q$ are demi-compact, then $\left\{\left(x_{n}, y_{n}\right)\right\}$ strongly converges to a solution of problem (1.9).

On taking $B=I$ and $H_{2}=H_{3}$ in Corollary 3.1, we obtain the following convergence theorem for the split feasibility problem (1.8).

Corollary 3.2 Let $H_{1}$ and $H_{2}$ be real Hilbert spaces, $C \subseteq H_{1}$ and $Q \subseteq H_{2}$ be nonempty closed convex subsets of $H_{1}$ and $H_{2}$, respectively. Let $P: H_{1} \longrightarrow H_{1}$ and $Q: H_{2} \longrightarrow H_{2}$ be two demi-contractive mappings with constants $k_{1}$ and $k_{2}$, respectively, with the condition $k \in(0,1)$, where $k=\max \left\{k_{1}, k_{2}\right\}$ such that $(I-P)$ and $(I-Q)$ are demi-closed at zero, and $\operatorname{Fix}(P) \neq \emptyset$ and $\operatorname{Fix}(Q) \neq \emptyset$. Let $A: H_{1} \longrightarrow H_{3}$ be a bounded linear mapping. Assume that $\left(x_{1}, y_{1}\right) \in C \times Q$ and the iteration scheme $\left\{\left(x_{n}, y_{n}\right)\right\}$ is defined as follows:

$$
\left\{\begin{array}{l}
x_{n+1}=\left(1-\alpha_{n}\right)\left(x_{n}-\gamma_{n} A^{*}\left(A x_{n}-y_{n}\right)\right)+\alpha_{n} P\left(x_{n}-\gamma_{n} A^{*}\left(A x_{n}-y_{n}\right)\right) \\
y_{n+1}=\left(1-\alpha_{n}\right)\left(y_{n}+\gamma_{n}\left(A x_{n}-y_{n}\right)\right)+\alpha_{n} Q\left(y_{n}+\gamma_{n}\left(A x_{n}-y_{n}\right)\right)
\end{array}\right.
$$

where $\lambda_{A}$ denotes the spectral radii of $A^{*} A,\left\{\gamma_{n}\right\}$ is a positive real sequence such that $\gamma_{n} \in$ $\left(\epsilon, \frac{1}{\lambda_{A}}-\epsilon\right)$ (for $\epsilon$ small enough), $\left\{\alpha_{n}\right\}$ is a sequence in $(k, 1)$ such that for some $\alpha, \beta \in(0,1)$, $0<\alpha \leq \alpha_{n} \leq \beta<1$.

If $\Omega:=\operatorname{Fix}(P) \cap \operatorname{Fix}(Q) \cap \operatorname{SFP} \neq \emptyset$, then:

(I) The sequence $\left\{\left(x_{n}, y_{n}\right)\right\}$ weakly converges to a solution of problem (1.8).

(II) In addition, if $P$ and $Q$ are demi-compact, then $\left\{\left(x_{n}, y_{n}\right)\right\}$ strongly converges to a solution of problem (1.8).

\section{Applications}

\subsection{Application to the split equality convex differentiable optimization problem}

The familiar problem

$$
\left\{\begin{array}{l}
\operatorname{minimize} \psi(x) \\
\text { subject to } x \in C,
\end{array}\right.
$$


in which $\psi: C \longrightarrow C$ is convex and differentiable has a special optimality criterion. A vector $x^{*}$ solves (4.1) if and only if it solves the following variational inequality problem: find $x^{*} \in C$ such that

$$
\left\langle\nabla \psi\left(x^{*}\right), y-x^{*}\right\rangle \geq 0, \quad \forall y \in C
$$

By putting $F\left(x^{*}, y\right)=\left\langle\nabla \psi\left(x^{*}\right), y-x^{*}\right\rangle$ in (1.1), we see that the variational inequality problem (4.2) and the equilibrium problem (1.1) have the same set of solutions.

In 2015, Ma et al. [15] introduced the so-called split equality mixed variational inequality problem which is finding $x^{*} \in C$ and $y^{*} \in Q$ such that

$$
\begin{aligned}
& \left\langle\psi\left(x^{*}\right), x-x^{*}\right\rangle+\phi(x)-\phi\left(x^{*}\right) \geq 0, \quad \forall x \in C, \\
& \left\langle\zeta\left(y^{*}\right), y-y^{*}\right\rangle+\varphi(y)-\varphi\left(y^{*}\right) \geq 0, \quad \forall y \in Q, \quad \text { and } \\
& A x^{*}=B y^{*},
\end{aligned}
$$

where $\psi: C \longrightarrow H_{1}$ and $\zeta: Q \longrightarrow H_{2}$ are the mappings.

The split equality mixed convex differentiable optimization problem can be viewed as analogous to the problem of finding $x^{*} \in C$ and $y^{*} \in Q$ such that

$$
\begin{aligned}
& \left\langle\nabla \psi\left(x^{*}\right), x-x^{*}\right\rangle+\left\langle T\left(x^{*}\right), x-x^{*}\right\rangle+\phi(x)-\phi\left(x^{*}\right) \geq 0, \quad \forall x \in C, \\
& \left\langle\nabla \zeta\left(y^{*}\right), y-y^{*}\right\rangle+\left\langle S\left(y^{*}\right), y-y^{*}\right\rangle+\varphi(y)-\varphi\left(y^{*}\right) \geq 0, \quad \forall y \in Q, \quad \text { and } \\
& A x^{*}=B y^{*},
\end{aligned}
$$

where $\psi: C \longrightarrow H_{1}$ and $\zeta: Q \longrightarrow H_{2}$ are convex and differentiable mappings. The set of solutions of the split equality mixed convex differentiable optimization problem (4.3) is denoted by $\operatorname{SEMCDOP}(\psi, \zeta, T, S, \phi, \varphi)$.

If $B=I$ and $H_{2}=H_{3}$, then the split mixed convex differentiable optimization problem can be viewed as analogous to the problem of finding $x^{*} \in C$ such that

$$
\left\langle\nabla \psi\left(x^{*}\right), x-x^{*}\right\rangle+\left\langle T\left(x^{*}\right), x-x^{*}\right\rangle+\phi(x)-\phi\left(x^{*}\right) \geq 0, \quad \forall x \in C,
$$

and such that $y^{*}=A x^{*} \in Q$ solves

$$
\left\langle\nabla \zeta\left(y^{*}\right), y-y^{*}\right\rangle+\left\langle S\left(y^{*}\right), y-y^{*}\right\rangle+\varphi(y)-\varphi\left(y^{*}\right) \geq 0, \quad \forall y \in Q
$$

The set of solutions of the split mixed convex differentiable optimization problem (4.4) is denoted by $\operatorname{SMCDOP}(\psi, \zeta, T, S, \phi, \varphi)$.

By setting $F\left(x^{*}, x\right)=\left\langle\nabla \psi\left(x^{*}\right), x-x^{*}\right\rangle$ and $G\left(y^{*}, y\right)=\left\langle\nabla \zeta\left(y^{*}\right), y-y^{*}\right\rangle$, for $\lambda_{1}=\lambda_{2}=1$, it is easy to see that $F$ and $G$ satisfy all the conditions (A1)-(A7) since the gradients $\nabla \psi$ and $\nabla \zeta$ are monotone mappings due to convexity and differentiability of $\psi$ and $\zeta$, respectively. Then from Theorem 3.1, we have the following result.

Theorem 4.1 Let $H_{1}, H_{2}$, and $H_{3}$ be real Hilbert spaces, $C \subseteq H_{1}$ and $Q \subseteq H_{2}$ be nonempty closed convex subsets of $H_{1}$ and $H_{2}$, respectively. Suppose that the mappings $\psi: C \rightarrow H_{1}$ and $\zeta: Q \longrightarrow H_{2}$ are convex and differentiable with optimality criterion, and the mappings 
$T: C \longrightarrow C$ and $S: Q \longrightarrow Q$ satisfy the conditions (A3), (A6), (A7). Let $\phi: C \longrightarrow \mathbb{R} \cup\{+\infty\}$ and $\varphi: Q \longrightarrow \mathbb{R} \cup\{+\infty\}$ be proper lower semicontinuous and convex mappings such that $C \cap \operatorname{dom} \phi \neq \emptyset$ and $Q \cap \operatorname{dom} \varphi \neq \emptyset$. Let $P: H_{1} \longrightarrow H_{1}$ and $Q: H_{2} \longrightarrow H_{2}$ be two demicontractive mappings with constants $k_{1}$ and $k_{2}$, respectively, with the condition $k \in(0,1)$, where $k=\max \left\{k_{1}, k_{2}\right\}$ such that $(I-P)$ and $(I-Q)$ are demi-closed at zero, and $\operatorname{Fix}(P) \neq \emptyset$ and $\operatorname{Fix}(Q) \neq \emptyset$. Let $A: H_{1} \longrightarrow H_{3}$ and $B: H_{2} \longrightarrow H_{3}$ be bounded linear mappings. Assume that $\left(x_{1}, y_{1}\right) \in C \times Q$ and the iteration scheme $\left\{\left(x_{n}, y_{n}\right)\right\}$ is defined as follows:

$$
\left\{\begin{array}{l}
\left\langle\nabla \psi\left(u_{n}\right), u-u_{n}\right\rangle+\left\langle T\left(u_{n}\right), u-u_{n}\right\rangle+\phi(u)-\phi\left(u_{n}\right)+\frac{1}{r_{n}}\left\langle u-u_{n}, u_{n}-x_{n}\right\rangle \geq 0 \\
\left\langle\nabla \zeta\left(v_{n}\right), v-v_{n}\right\rangle+\left\langle S\left(v_{n}\right), v-v_{n}\right\rangle+\varphi(v)-\varphi\left(v_{n}\right)+\frac{1}{r_{n}}\left\langle v-v_{n}, v_{n}-y_{n}\right\rangle \geq 0 ; \\
x_{n+1}=\left(1-\alpha_{n}\right)\left(u_{n}-\gamma_{n} A^{*}\left(A u_{n}-B v_{n}\right)\right)+\alpha_{n} P\left(u_{n}-\gamma_{n} A^{*}\left(A u_{n}-B v_{n}\right)\right) ; \\
y_{n+1}=\left(1-\alpha_{n}\right)\left(v_{n}+\gamma_{n} B^{*}\left(A u_{n}-B v_{n}\right)\right)+\alpha_{n} Q\left(v_{n}+\gamma_{n} B^{*}\left(A u_{n}-B v_{n}\right)\right),
\end{array}\right.
$$

for every $u \in C, v \in Q$ and $n \geq 1$ where $\lambda_{A}$ and $\lambda_{B}$ denote the spectral radii of $A^{*} A$ and $B^{*} B$, respectively, $\left\{\gamma_{n}\right\}$ is a positive real sequence such that $\gamma_{n} \in\left(\epsilon, \frac{2}{\lambda_{A}+\lambda_{B}}-\epsilon\right)$ (for $\epsilon$ small enough), $\left\{\alpha_{n}\right\}$ is a sequence in $(k, 1)$, and $\left\{r_{n}\right\} \subset(0, \infty)$ satisfying the following conditions:

(i) for some $\alpha, \beta \in(0,1), 0<\alpha \leq \alpha_{n} \leq \beta<1$;

(ii) $\liminf _{n \rightarrow \infty} r_{n}>0$ and $\lim _{n \rightarrow \infty}\left|r_{n+1}-r_{n}\right|=0$.

If $\Omega:=\operatorname{Fix}(P) \cap \operatorname{Fix}(Q) \cap \operatorname{SEMCDOP}(\psi, \zeta, T, S, \phi, \varphi) \neq \emptyset$, then:

(I) The sequence $\left\{\left(x_{n}, y_{n}\right)\right\}$ weakly converges to a solution of problem (4.3).

(II) In addition, if $P$ and $Q$ are demi-compact, then $\left\{\left(x_{n}, y_{n}\right)\right\}$ strongly converges to a solution of problem (4.3).

If we take $B=I$ and $H_{2}=H_{3}$ in Theorem 4.1, then we have the following convergence result for the split mixed convex differentiable optimization problem (4.4).

Corollary 4.1 Let $H_{1}$ and $H_{2}$ be real Hilbert spaces, $C \subseteq H_{1}$ and $Q \subseteq H_{2}$ be nonempty closed convex subsets of $H_{1}$ and $H_{2}$, respectively. Suppose that the mappings $\psi: C \rightarrow H_{1}$ and $\zeta: Q \longrightarrow H_{2}$ are convex and differentiable with optimality criterion, and the mappings $T: C \longrightarrow C$ and $S: Q \longrightarrow Q$ satisfy the conditions (A3), (A6), (A7). Let $\phi: C \longrightarrow \mathbb{R} \cup\{+\infty\}$ and $\varphi: Q \rightarrow \mathbb{R} \cup\{+\infty\}$ be proper lower semicontinuous and convex mappings such that $C \cap \operatorname{dom} \phi \neq \emptyset$ and $Q \cap \operatorname{dom} \varphi \neq \emptyset$. Let $P: H_{1} \longrightarrow H_{1}$ and $Q: H_{2} \longrightarrow H_{2}$ be two demicontractive mappings with constants $k_{1}$ and $k_{2}$, respectively, with the condition $k \in(0,1)$, where $k=\max \left\{k_{1}, k_{2}\right\}$ such that $(I-P)$ and $(I-Q)$ are demi-closed at zero, and $\operatorname{Fix}(P) \neq \emptyset$ and $\operatorname{Fix}(Q) \neq \emptyset$. Let $A: H_{1} \longrightarrow H_{3}$ be a bounded linear mapping. Assume that $\left(x_{1}, y_{1}\right) \in$ $C \times Q$ and the iteration scheme $\left\{\left(x_{n}, y_{n}\right)\right\}$ is defined as follows:

$$
\left\{\begin{array}{l}
\left\langle\nabla \psi\left(u_{n}\right), u-u_{n}\right\rangle+\left\langle T\left(u_{n}\right), u-u_{n}\right\rangle+\phi(u)-\phi\left(u_{n}\right)+\frac{1}{r_{n}}\left\langle u-u_{n}, u_{n}-x_{n}\right\rangle \geq 0 ; \\
\left\langle\nabla \zeta\left(v_{n}\right), v-v_{n}\right\rangle+\left\langle S\left(v_{n}\right), v-v_{n}\right\rangle+\varphi(v)-\varphi\left(v_{n}\right)+\frac{1}{r_{n}}\left\langle v-v_{n}, v_{n}-y_{n}\right\rangle \geq 0 ; \\
x_{n+1}=\left(1-\alpha_{n}\right)\left(u_{n}-\gamma_{n} A^{*}\left(A u_{n}-v_{n}\right)\right)+\alpha_{n} P\left(u_{n}-\gamma_{n} A^{*}\left(A u_{n}-v_{n}\right)\right) ; \\
y_{n+1}=\left(1-\alpha_{n}\right)\left(v_{n}+\gamma_{n}\left(A u_{n}-v_{n}\right)\right)+\alpha_{n} Q\left(v_{n}+\gamma_{n}\left(A u_{n}-v_{n}\right)\right),
\end{array}\right.
$$

for every $u \in C, v \in Q$, and $n \geq 1$ where $\lambda_{A}$ denotes the spectral radius of $A^{*} A,\left\{\gamma_{n}\right\}$ is a positive real sequence such that $\gamma_{n} \in\left(\epsilon, \frac{1}{\lambda_{A}}-\epsilon\right)$ (for $\epsilon$ small enough), $\left\{\alpha_{n}\right\}$ is a sequence in $(k, 1)$, and $\left\{r_{n}\right\} \subset(0, \infty)$ satisfying the following conditions: 
(i) for some $\alpha, \beta \in(0,1), 0<\alpha \leq \alpha_{n} \leq \beta<1$;

(ii) $\liminf _{n \rightarrow \infty} r_{n}>0$ and $\lim _{n \rightarrow \infty}\left|r_{n+1}-r_{n}\right|=0$.

If $\Omega:=\operatorname{Fix}(P) \cap \operatorname{Fix}(Q) \cap \operatorname{SMCDOP}(\psi, \zeta, T, S, \phi, \varphi) \neq \emptyset$, then:

(I) The sequence $\left\{\left(x_{n}, y_{n}\right)\right\}$ weakly converges to a solution of problem (4.4).

(II) In addition, if $P$ and $Q$ are demi-compact, then $\left\{\left(x_{n}, y_{n}\right)\right\}$ strongly converges to a solution of problem (4.4).

\subsection{Application to the split equality convex minimization problem}

Problem (1.19) is called a split equality convex minimization problem. From Theorem 3.1, we can conclude to the following convergence result for problem (1.19).

Theorem 4.2 Let $H_{1}, H_{2}$, and $H_{3}$ be real Hilbert spaces, $C \subseteq H_{1}$ and $Q \subseteq H_{2}$ be nonempty closed convex subsets of $H_{1}$ and $H_{2}$, respectively. Let $\phi: C \longrightarrow \mathbb{R} \cup\{+\infty\}$ and $\varphi: Q \longrightarrow$ $\mathbb{R} \cup\{+\infty\}$ be proper lower semicontinuous and convex mappings such that $C \cap \operatorname{dom} \phi \neq \emptyset$ and $Q \cap \operatorname{dom} \varphi \neq \emptyset$. Let $P: H_{1} \longrightarrow H_{1}$ and $Q: H_{2} \longrightarrow H_{2}$ be two demi-contractive mappings with constants $k_{1}$ and $k_{2}$, respectively, with the condition $k \in(0,1)$, where $k=\max \left\{k_{1}, k_{2}\right\}$ such that $(I-P)$ and $(I-Q)$ are demi-closed at zero, and $\operatorname{Fix}(P) \neq \emptyset$ and $\operatorname{Fix}(Q) \neq \emptyset$. Let $A: H_{1} \longrightarrow H_{3}$ and $B: H_{2} \longrightarrow H_{3}$ be bounded linear mappings. Assume that $\left(x_{1}, y_{1}\right) \in C \times Q$ and the iteration scheme $\left\{\left(x_{n}, y_{n}\right)\right\}$ is defined as follows:

$$
\left\{\begin{array}{l}
\phi(u)-\phi\left(u_{n}\right)+\frac{1}{r_{n}}\left\langle u-u_{n}, u_{n}-x_{n}\right\rangle \geq 0, \quad \forall u \in C ; \\
\varphi(v)-\varphi\left(v_{n}\right)+\frac{1}{r_{n}}\left\langle v-v_{n}, v_{n}-y_{n}\right\rangle \geq 0, \quad \forall v \in Q ; \\
x_{n+1}=\left(1-\alpha_{n}\right)\left(u_{n}-\gamma_{n} A^{*}\left(A u_{n}-B v_{n}\right)\right)+\alpha_{n} P\left(u_{n}-\gamma_{n} A^{*}\left(A u_{n}-B v_{n}\right)\right) ; \\
y_{n+1}=\left(1-\alpha_{n}\right)\left(v_{n}+\gamma_{n} B^{*}\left(A u_{n}-B v_{n}\right)\right)+\alpha_{n} Q\left(v_{n}+\gamma_{n} B^{*}\left(A u_{n}-B v_{n}\right)\right), \quad n \geq 1,
\end{array}\right.
$$

where $\lambda_{A}$ and $\lambda_{B}$ denote the spectral radii of $A^{*} A$ and $B^{*} B$, respectively, $\left\{\gamma_{n}\right\}$ is a positive real sequence such that $\gamma_{n} \in\left(\epsilon, \frac{2}{\lambda_{A}+\lambda_{B}}-\epsilon\right)$ (for $\epsilon$ small enough), $\left\{\alpha_{n}\right\}$ is a sequence in $(k, 1)$, and $\left\{r_{n}\right\} \subset(0, \infty)$ satisfying the following conditions:

(i) for some $\alpha, \beta \in(0,1), 0<\alpha \leq \alpha_{n} \leq \beta<1$;

(ii) $\liminf _{n \rightarrow \infty} r_{n}>0$ and $\lim _{n \rightarrow \infty}\left|r_{n+1}-r_{n}\right|=0$.

If $\Omega:=\operatorname{Fix}(P) \cap \operatorname{Fix}(Q) \cap \operatorname{SECMP}(\phi, \varphi) \neq \emptyset$, then:

(I) The sequence $\left\{\left(x_{n}, y_{n}\right)\right\}$ weakly converges to a solution of problem (1.19).

(II) In addition, if $P$ and $Q$ are demi-compact, then $\left\{\left(x_{n}, y_{n}\right)\right\}$ strongly converges to a solution of problem (1.19).

If we take $B=I$ and $H_{2}=H_{3}$ in Theorem 4.2, then we have the following convergence result for the split convex minimization problem (1.20).

Corollary 4.2 Let $H_{1}$ and $H_{2}$ be real Hilbert spaces, $C \subseteq H_{1}$ and $Q \subseteq H_{2}$ be nonempty closed convex subsets of $H_{1}$ and $H_{2}$, respectively. Let $\phi: C \longrightarrow \mathbb{R} \cup\{+\infty\}$ and $\varphi: Q \longrightarrow$ $\mathbb{R} \cup\{+\infty\}$ be proper lower semicontinuous and convex mappings such that $C \cap \operatorname{dom} \phi \neq \emptyset$ and $Q \cap \operatorname{dom} \varphi \neq \emptyset$. Let $P: H_{1} \longrightarrow H_{1}$ and $Q: H_{2} \longrightarrow H_{2}$ be two demi-contractive mappings with constants $k_{1}$ and $k_{2}$, respectively, with the condition $k \in(0,1)$, where $k=\max \left\{k_{1}, k_{2}\right\}$ such that $(I-P)$ and $(I-Q)$ are demi-closed at zero, and $\operatorname{Fix}(P) \neq \emptyset$ and $\operatorname{Fix}(Q) \neq \emptyset$. Let $A: H_{1} \longrightarrow H_{3}$ be a bounded linear mapping. Assume that $\left(x_{1}, y_{1}\right) \in C \times Q$ and the iteration 
scheme $\left\{\left(x_{n}, y_{n}\right)\right\}$ is defined as follows:

$$
\left\{\begin{array}{l}
\phi(u)-\phi\left(u_{n}\right)+\frac{1}{r_{n}}\left\langle u-u_{n}, u_{n}-x_{n}\right\rangle \geq 0, \quad \forall u \in C ; \\
\varphi(v)-\varphi\left(v_{n}\right)+\frac{1}{r_{n}}\left\langle v-v_{n}, v_{n}-y_{n}\right\rangle \geq 0, \quad \forall v \in Q ; \\
x_{n+1}=\left(1-\alpha_{n}\right)\left(u_{n}-\gamma_{n} A^{*}\left(A u_{n}-B v_{n}\right)\right)+\alpha_{n} P\left(u_{n}-\gamma_{n} A^{*}\left(A u_{n}-B v_{n}\right)\right) ; \\
y_{n+1}=\left(1-\alpha_{n}\right)\left(v_{n}+\gamma_{n} B^{*}\left(A u_{n}-B v_{n}\right)\right)+\alpha_{n} Q\left(v_{n}+\gamma_{n} B^{*}\left(A u_{n}-B v_{n}\right)\right), \quad n \geq 1,
\end{array}\right.
$$

where $\lambda_{A}$ denotes the spectral radii of $A^{*} A,\left\{\gamma_{n}\right\}$ is a positive real sequence such that $\gamma_{n} \in$ $\left(\epsilon, \frac{1}{\lambda_{A}}-\epsilon\right)$ (for $\epsilon$ small enough), $\left\{\alpha_{n}\right\}$ is a sequence in $(k, 1)$, and $\left\{r_{n}\right\} \subset(0, \infty)$ satisfying the following conditions:

(i) for some $\alpha, \beta \in(0,1), 0<\alpha \leq \alpha_{n} \leq \beta<1$;

(ii) $\liminf _{n \rightarrow \infty} r_{n}>0$ and $\lim _{n \rightarrow \infty}\left|r_{n+1}-r_{n}\right|=0$.

If $\Omega:=\operatorname{Fix}(P) \cap \operatorname{Fix}(Q) \cap \operatorname{SCMP}(\phi, \varphi) \neq \emptyset$, then:

(I) The sequence $\left\{\left(x_{n}, y_{n}\right)\right\}$ weakly converges to a solution of problem (1.20).

(II) In addition, if $P$ and $Q$ are demi-compact, then $\left\{\left(x_{n}, y_{n}\right)\right\}$ strongly converges to a solution of problem (1.20).

\subsection{Application to the split equality mixed equilibrium problem}

It is easy to see that on taking $\lambda_{1}=\lambda_{2}=1$ and $T=S=0$ in problem (1.17), then the split equality generalized mixed equilibrium problem (1.17) becomes the split equality mixed equilibrium problem (1.15), which was considered by Ma et al. [15]. The following is the convergence result for problem (1.15).

Theorem 4.3 Let $H_{1}, H_{2}$, and $H_{3}$ be real Hilbert spaces, $C \subseteq H_{1}$ and $Q \subseteq H_{2}$ be nonempty closed convex subsets of $\mathrm{H}_{1}$ and $\mathrm{H}_{2}$, respectively. Suppose that the bi-mappings $F: C \times$ $C \rightarrow \mathbb{R}$ and $G: Q \times Q \rightarrow \mathbb{R}$ satisfy the conditions (A1), (A2), (A4), (A5), and (A7). Let $\phi: C \longrightarrow \mathbb{R} \cup\{+\infty\}$ and $\varphi: Q \rightarrow \mathbb{R} \cup\{+\infty\}$ be proper lower semicontinuous and convex mappings such that $C \cap \operatorname{dom} \phi \neq \emptyset$ and $Q \cap \operatorname{dom} \varphi \neq \emptyset$. Let $P: H_{1} \longrightarrow H_{1}$ and $Q: H_{2} \longrightarrow H_{2}$ be two demi-contractive mappings with constants $k_{1}$ and $k_{2}$, respectively, with the condition $k \in(0,1)$, where $k=\max \left\{k_{1}, k_{2}\right\}$ such that $(I-P)$ and $(I-Q)$ are demi-closed at zero, and $\operatorname{Fix}(P) \neq \emptyset$ and $\operatorname{Fix}(Q) \neq \emptyset$. Let $A: H_{1} \longrightarrow H_{3}$ and $B: H_{2} \longrightarrow H_{3}$ be bounded linear mappings. Assume that $\left(x_{1}, y_{1}\right) \in C \times Q$ and the iteration scheme $\left\{\left(x_{n}, y_{n}\right)\right\}$ is defined as follows:

$$
\left\{\begin{array}{l}
F\left(u_{n}, u\right)+\phi(u)-\phi\left(u_{n}\right)+\frac{1}{r_{n}}\left\langle u-u_{n}, u_{n}-x_{n}\right\rangle \geq 0, \quad \forall u \in C ; \\
G\left(v_{n}, v\right)+\varphi(v)-\varphi\left(v_{n}\right)+\frac{1}{r_{n}}\left\langle v-v_{n}, v_{n}-y_{n}\right\rangle \geq 0, \quad \forall v \in Q ; \\
x_{n+1}=\left(1-\alpha_{n}\right)\left(u_{n}-\gamma_{n} A^{*}\left(A u_{n}-B v_{n}\right)\right)+\alpha_{n} P\left(u_{n}-\gamma_{n} A^{*}\left(A u_{n}-B v_{n}\right)\right) ; \\
y_{n+1}=\left(1-\alpha_{n}\right)\left(v_{n}+\gamma_{n} B^{*}\left(A u_{n}-B v_{n}\right)\right)+\alpha_{n} Q\left(v_{n}+\gamma_{n} B^{*}\left(A u_{n}-B v_{n}\right)\right), \quad n \geq 1,
\end{array}\right.
$$

where $\lambda_{A}$ and $\lambda_{B}$ denote the spectral radii of $A^{*} A$ and $B^{*} B$, respectively, $\left\{\gamma_{n}\right\}$ is a positive real sequence such that $\gamma_{n} \in\left(\epsilon, \frac{2}{\lambda_{A}+\lambda_{B}}-\epsilon\right)$ (for $\epsilon$ small enough), $\left\{\alpha_{n}\right\}$ is a sequence in $(k, 1)$, and $\left\{r_{n}\right\} \subset(0, \infty)$ satisfying the following conditions:

(i) for some $\alpha, \beta \in(0,1), 0<\alpha \leq \alpha_{n} \leq \beta<1$;

(ii) $\liminf _{n \rightarrow \infty} r_{n}>0$ and $\lim _{n \rightarrow \infty}\left|r_{n+1}-r_{n}\right|=0$.

If $\Omega:=\operatorname{Fix}(P) \cap \operatorname{Fix}(Q) \cap \operatorname{SEMEP}(F, G, \phi, \varphi) \neq \emptyset$, then: 
(I) The sequence $\left\{\left(x_{n}, y_{n}\right)\right\}$ weakly converges to a solution of problem (1.15).

(II) In addition, if $P$ and $Q$ are demi-compact, then $\left\{\left(x_{n}, y_{n}\right)\right\}$ strongly converges to a solution of problem (1.15).

\section{Competing interests}

The authors declare that they have no competing interests.

\section{Authors' contributions}

The authors contributed equally and significantly in writing this paper. All authors read and approved the final manuscript.

\section{Author details}

'Department of Mathematics, Aligarh Muslim University, Aligarh, 202002, India. ²Department of Information Management, Cheng Shiu University, Kaohsiung, 833, Taiwan. ${ }^{3}$ Research Center of Nonlinear Analysis and Optimization and Center for Fundamental Science, Kaohsiung Medical University, Kaohsiung, 807, Taiwan.

\section{Acknowledgements}

YCL is supported in part by the following projects with grant no. MOST 103-2923-E-037-001-MY3, MOST

104-2221-E-230-004 and Kaohsiung Medical University 'Aim for the Top Universities Grant, grant no. KMU-TP103F00'. This research is partially supported by the grants of MOST 101-2628-E-230-001-MY3 and MOST 101-2622-E-230-005-CC3.

\section{Received: 14 August 2015 Accepted: 8 December 2015 Published online: 30 December 2015}

\section{References}

1. Blum, E, Oettli, W: From optimization and variational inequalities to equilibrium problems. Math. Stud. 63, 123-145 (1994)

2. Ahmad, R, Rahaman, M: Generalized strongly vector equilibrium problem for set-valued mappings. Filomat 28(9), 1783-1790 (2014)

3. Moudafi, A, Thèra, M: Proximal and dynamical approaches to equilibrium problems. In: III-posed Variational Problems and Regularization Techniques. Lecture Notes in Economics and Mathematical Systems, vol. 477, pp. 187-201. Springer, New York (1999)

4. Peng, J-W, Yao, J-C: A new hybrid-extragradient method for generalized mixed equilibrium problems, fixed point problems and variational inequality problems. Taiwan. J. Math. 12(6), 1401-1432 (2008)

5. Ceng, L-C, Yao, J-C: A hybrid iterative scheme for mixed equilibrium problems and fixed point problems. J. Comput. Appl. Math. 214, 186-201 (2008)

6. Chang, AS, Yao, J-C, Kim, JJ, Yang, L: Iterative approximation to convex feasibility problems in Banach space. Fixed Point Theory Appl. 2007, 46797 (2007). doi:10.1155/2007/46797

7. Byrne, C: Iterative oblique projection onto convex subsets and the split feasibility problem. Inverse Probl. 18, 441-453 (2002)

8. Censor, Y, Bortfeld, T, Martin, N, Trofimov, A: A unified approach for inversion problem in intensity-modulated radiation therapy. Phys. Med. Biol. 51, 2353-2365 (2006)

9. Censor, Y, Elfving, T, Kopf, N, Bortfeld, T: The multiple-sets split feasibility problem and its applications for inverse problems. Inverse Probl. 21, 2071-2084 (2005)

10. Censor, Y, Elfving, T: A multiprojection algorithm using Bregman projections in a product space. Numer. Algorithms 8(2), 221-239 (1994)

11. Palta, RJ, Mackie, TR (eds.): Intensity-Modulated Radiation Therapy: The State of Art. Medical Physics Monograph. Medical Physics Publishing, Madison (2003)

12. Moudafi, A, Al-Shemas, E: Simultaneous iterative methods for split equality problems. Trans. Math. Program. Appl. 1(2), 1-11 (2013)

13. Ma, Y-F, Wang, L, Zi, X-J: Strong and weak convergence theorems for a new split feasibility problem. Int. Math. Forum 8(33), 1621-1627 (2013)

14. He, Z: The split equilibrium problem and its convergence algorithms. J. Inequal. Appl. 2012, 162 (2012). doi:10.1186/1029-242X-2012-162

15. Ma, Z, Wang, L, Chang, S-S, Duan, W: Convergence theorems for split equality mixed equilibrium problems with applications. Fixed Point Theory Appl. 2015, 31 (2015). doi:10.1186/s13663-015-0281-x

16. Chidume, CE, Ndambomve, P, Bello, AU, Okpala, ME: The multiple-sets split equality fixed point problem for countable families of multi-valued demi-contractive mappings. Int. J. Math. Anal. 9(10), 453-469 (2015)

17. Fan, K: A generalization of Tychonoff's fixed point theorem. Math. Ann. 142, 305-310 (1961)

18. Goebel, K, Reich, S: Uniform Convexity, Hyperbolic Geometry and Nonexpansive Mappings. Dekker, New York (1984)

19. Opial, Z: Weak convergence of the sequence of successive approximations for nonexpansive mappings. Bull. Am. Math. Soc. 73, 591-597 (1967)

20. Marino, G, Xu, HK: Weak and strong convergence theorems for strict pseudocontractions in Hilbert space. J. Math. Anal. Appl. 329, 336-346 (2007) 